\title{
Sigmoidal-trapezoidal quadrature for ordinary and Cauchy principal value integrals
}

\author{
David Elliott*
}

(Received 6 October 2003; revised 8 June 2004)

\begin{abstract}
Consider the evaluation of If $:=\int_{0}^{1} f(x) d x$. Among all the quadrature rules for the approximate evaluation of this integral, the trapezoidal rule is known for its simplicity of construction and, in general, its slow rate of convergence to $I f$. However, it is well known, from the Euler-Maclaurin formula, that if $f$ is periodic of period 1, then the trapezoidal rule can converge very quickly to $I f$. A sigmoidal transformation is a mapping of $[0,1]$ onto itself and is such that when applied to If gives an integrand having some degree of periodicity. Applying the trapezoidal rule to the transformed integral gives an increased rate of convergence. In this paper, we explore the use of such transformations for both ordinary and Cauchy principal value integrals. By considering the problem in a suitably weighted Sobolev
\end{abstract}

*School of Mathematics and Physics, University of Tasmania, Private Bag 37, Hobart, Tasmania 7001, AustraliA. mailto:elliott@hilbert.maths.utas.edu.au

See http://anziamj.austms.org.au/V46/E/Elliott/home.html for this article, (C) Austral. Mathematical Soc. 2004. Published July 17, 2004. ISSN 1446-8735 
space, a very satisfactory analysis of the rate of convergence of the truncation error is obtained. This combination of a sigmoidal transformation followed by the trapezoidal rule gives rise to the so-called sigmoidal-trapezoidal quadrature rule of the title.

\section{Contents}

1 Introduction

E3

2 A weighted Sobolev space

E3

3 Sigmoidal transformations

E24

4 The Euler-Maclaurin formula

E29

5 Cauchy Principal Value Integrals

E38

5.1 Introduction . . . . . . . . . . . . . . . . E38

5.2 The subtraction function . . . . . . . . . . . . E39

5.3 The function $f_{n} \ldots \ldots \ldots \ldots \ldots$ E46

5.4 The denouement . . . . . . . . . . . . . E54

5.5 Sigmoidal transformations . . . . . . . . . . . E57

6 Conclusion

E61

A Appendix

E62

References

E68 


\section{Introduction}

In a recent paper [4], I discussed the Euler-Maclaurin formula for the approximate evaluation of the integral $\int_{0}^{1} f(x) d x$, after introducing a sigmoidal transformation which maps $[0,1]$ onto itself. (For a discussion on sigmoidal transformations, see [5].) In [4] I gave an error analysis based on a space of functions introduced by Kress [7]. The purpose of this paper is to introduce a weighted Sobolev space, which is more general than the space given by Kress and which enables us both to extend and improve some of the truncation error estimates previously given.

\section{A weighted Sobolev space}

Throughout the paper we let $\Omega$ denote the open interval $(0,1)$ with $\bar{\Omega}$ denoting the closed interval $[0,1]$. We let $\mathbb{N}_{0}$ denote the set of all non-negative integers, that is, $\mathbb{N}_{0}:=\{0,1,2, \ldots\}$ and $\mathbb{N}$ denotes the set of all positive integers. We also write $\mathbb{Z}$ to denote the set of all integers. The notation $k=0(1) N$ means that $k$ takes all values in the set $\{0,1,2, \ldots, N\}$. We now introduce the weighted Sobolev space $W_{p, \alpha}^{(N)}(\Omega)$ where $1 \leq p \leq \infty, N \in \mathbb{N}_{0}$ and $\alpha \in \mathbb{R}$, the set of all real numbers.

Definition 1 (a) For $1 \leq p<\infty, \alpha \in \mathbb{R}$ and $N \in \mathbb{N}_{0}$ we say that $f$ is in the weighted Sobolev space $W_{p, \alpha}^{(N)}(\Omega)$ if

$$
\int_{0}^{1}\left[(t(1-t))^{k-\alpha}\left|f^{(k)}(t)\right|\right]^{p} d t<\infty
$$

for $k=0(1) N$, in which case the norm of $f$ will be denoted and defined by

$$
\|f\|_{p, \alpha, N}:=\max _{k=0(1) N}\left\{\int_{0}^{1}\left[(t(1-t))^{k-\alpha}\left|f^{(k)}(t)\right|\right]^{p} d t\right\}^{1 / p}
$$


(b) For $p=\infty, \alpha \in \mathbb{R}$ and $N \in \mathbb{N}_{0}$ we say that $f$ is in the weighted Sobolev space $W_{\infty, \alpha}^{(N)}(\Omega)$ if

$$
f^{(k)} \in C(\Omega) \quad \text { and } \quad \max _{t \in \bar{\Omega}}(t(1-t))^{k-\alpha}\left|f^{(k)}(t)\right|<\infty,
$$

for $k=0(1) N$. The norm of $f$ is denoted and defined by

$$
\|f\|_{\infty, \alpha, N}:=\max _{k=0(1) N} \max _{t \in \bar{\Omega}}(t(1-t))^{k-\alpha}\left|f^{(k)}(t)\right| .
$$

In this definition, $C(\Omega)$ denotes the space of all functions which are continuous on $\Omega=(0,1)$. Again, if $f \in C^{\infty}(\Omega)$ and if, for some $\alpha \in \mathbb{R}$ and $1 \leq p \leq \infty$, we have $f \in W_{p, \alpha}^{(N)}(\Omega)$ for every $N \in \mathbb{N}_{0}$ then we write $f \in W_{p, \alpha}^{(\infty)}(\Omega)$.

With this definition, let us consider some immediate consequences and in order to do this we shall make use of Hölder's inequality. For $1 \leq p, q \leq \infty$, we say that these are conjugate numbers if

$$
\frac{1}{p}+\frac{1}{q}=1
$$

Note that for $1<p<\infty$ we shall always have $1<q<\infty$. However, when $p=1$, we see from (3) that $1 / q=0$ so that $q=\infty$; similarly $q=1$ corresponds to $p=\infty$. Throughout the paper whenever we introduce $p$ we shall always assume that $q$ is its conjugate number satisfying (3). For $1<p<\infty$, Hölder's inequality states that for $f \in L_{p}(\Omega)$ and $g \in L_{q}(\Omega)$ then

$$
\left|\int_{0}^{1} f(t) g(t) d t\right| \leq\left(\int_{0}^{1}|f(t)|^{p} d t\right)^{1 / p}\left(\int_{0}^{1}|g(t)|^{q} d t\right)^{1 / q}=\|f\|_{p}\|g\|_{q} .
$$

When $p=1$ we again have (4) provided that we define $\|g\|_{\infty}$ as

$$
\|g\|_{\infty}:=\lim _{q \rightarrow \infty}\left(\int_{0}^{1}|g(t)|^{q} d t\right)^{1 / q}:=\operatorname{ess} \sup g
$$


where ess sup $g$ means the essential supremum of $g$. That is, if $|g(t)| \leq M$ almost everywhere on $\bar{\Omega}$, where $M$ is the smallest possible number for which this inequality is satisfied, then $\|g\|_{\infty}=M$. In the particular case when $g$ is continuous on $\bar{\Omega}$, then we have that $\|g\|_{\infty}=\max _{t \in \bar{\Omega}}|g(t)|$. Hölder's inequality in this case follows immediately from the generalized mean value theorem of integral calculus.

Theorem 2 (a) For $1 \leq p \leq \infty, \alpha \in \mathbb{R}$ and $N \in \mathbb{N}_{0}$ we have

$$
W_{\infty, \alpha}^{(N)}(\Omega) \subseteq W_{p, \alpha}^{(N)}(\Omega) \subseteq W_{1, \alpha}^{(N)}(\Omega),
$$

with

$$
\|f\|_{1, \alpha, N} \leq\|f\|_{p, \alpha, N} \leq\|f\|_{\infty, \alpha, N}
$$

(b) For $1 \leq p \leq \infty$ and $\alpha, \beta \in \mathbb{R}$ with $\beta \leq \alpha$ we have $W_{p, \alpha}^{(N)}(\Omega) \subseteq W_{p, \beta}^{(N)}(\Omega)$ with

$$
\|f\|_{p, \beta, N} \leq(1 / 4)^{(\alpha-\beta)}\|f\|_{p, \alpha, N}
$$

(c) If $f \in W_{p, \alpha}^{(N)}(\Omega)$ for $1 \leq p \leq \infty$, real $\alpha$ and $N \in \mathbb{N}$ then for $j=0(1) N$ we have $f^{(j)} \in W_{p, \alpha-j}^{(N-j)}(\Omega)$ with

$$
\left\|f^{(j)}\right\|_{p, \alpha-j, N-j} \leq\|f\|_{p, \alpha, N} .
$$

\section{Proof:}

(a) Suppose $f \in W_{\infty, \alpha}^{(N)}(\Omega)$ then for any $1 \leq p<\infty$ and $k \in\{0,1,2, \ldots, N\}$ we have

$$
\int_{0}^{1}\left[(t(1-t))^{k-\alpha}\left|f^{(k)}(t)\right|\right]^{p} d t \leq\left(\max _{t \in \bar{\Omega}}(t(1-t))^{k-\alpha}\left|f^{(k)}(t)\right|\right)^{p} \leq\|f\|_{\infty, \alpha, N}^{p} .
$$

Thus $f \in W_{p, \alpha}^{(N)}(\Omega)$ and we find

$$
\|f\|_{p, \alpha, N} \leq\|f\|_{\infty, \alpha, N} .
$$


Again, if $f \in W_{p, \alpha}^{(N)}(\Omega)$ for $1<p<\infty$ then, by Hölder's inequality (4), we have

$$
\begin{aligned}
& \int_{0}^{1}(t(1-t))^{k-\alpha}\left|f^{(k)}(t)\right| d t \\
\leq & \left(\int_{0}^{1}\left[(t(1-t))^{k-\alpha}\left|f^{(k)}(t)\right|\right]^{p} d t\right)^{1 / p}\left(\int_{0}^{1} 1^{q} d t\right)^{1 / q} \\
\leq & \|f\|_{p, \alpha, N} .
\end{aligned}
$$

On letting $k$ take all values in $\{0,1,2, \ldots, N\}$ in (11), we find

$$
\|f\|_{1, \alpha, N} \leq\|f\|_{p, \alpha, N}
$$

which, together with (10), establishes (a).

(b) Suppose that $1 \leq p<\infty$. For any $f \in W_{p, \alpha}^{(N)}(\Omega)$ we have

$$
\begin{aligned}
& \int_{0}^{1}\left[(t(1-t))^{k-\beta}\left|f^{(k)}(t)\right|\right]^{p} d t \\
& =\int_{0}^{1}\left[(t(1-t))^{k-\alpha}\left|f^{(k)}(t)\right|\right]^{p}(t(1-t))^{(\alpha-\beta) p} d t \\
& \leq(1 / 4)^{(\alpha-\beta) p}\|f\|_{p, \alpha, N}^{p},
\end{aligned}
$$

by the mean value theorem since $\alpha-\beta \geq 0$ so that $[t(1-t)]^{(\alpha-\beta) p}$ is continuous on $\bar{\Omega}$. Hence $W_{p, \alpha}^{(N)}(\Omega) \subseteq W_{p, \beta}^{(N)}(\Omega)$ and, on letting $k$ take all values in $\{0,1,2, \ldots, N\}$, from (12) we find

$$
\|f\|_{p, \beta, N} \leq(1 / 4)^{(\alpha-\beta)}\|f\|_{p, \alpha, N} .
$$

When $p=\infty$, if we suppose that $f \in W_{\infty, \alpha}^{(N)}(\Omega)$ we have

$$
\begin{aligned}
& \max _{t \in \bar{\Omega}}(t(1-t))^{k-\beta}\left|f^{(k)}(t)\right| \\
& =\max _{t \in \bar{\Omega}}(t(1-t))^{k-\alpha}\left|f^{(k)}(t)\right|(t(1-t))^{\alpha-\beta} \\
& \leq(1 / 4)^{(\alpha-\beta)}\|f\|_{\infty, \alpha, N} .
\end{aligned}
$$


Thus, arguing as before, $f \in W_{\infty, \beta}^{(N)}(\Omega)$ with

$$
\|f\|_{\infty, \beta, N} \leq(1 / 4)^{(\alpha-\beta)}\|f\|_{\infty, \alpha, N} .
$$

This proves (b).

(c) Suppose first that $1 \leq p<\infty$. Now $f \in W_{p, \alpha}^{(N)}(\Omega)$ implies that

$$
\int_{0}^{1}\left[(t(1-t))^{k-\alpha}\left|f^{(k)}(t)\right|\right]^{p} d t \leq\|f\|_{p, \alpha, N}^{p}
$$

Fix $j \in\{0,1,2, \ldots, N\}$ and write $k=l+j$ where $l \in\{0,1, \ldots, N-j\}$. Then we rewrite (14) as

$$
\int_{0}^{1}\left[(t(1-t))^{l-(\alpha-j)}\left|\left(f^{(j)}(t)\right)^{(l)}\right|\right]^{p} d t \leq\|f\|_{p, \alpha, N}^{p} .
$$

This implies $f^{(j)} \in W_{p, \alpha-j}^{(N-j)}(\Omega)$ and, further, $\left\|f^{(j)}\right\|_{p, \alpha-j, N-j} \leq\|f\|_{p, \alpha, N}$. When $p=\infty$ we have, from

$$
\max _{t \in \bar{\Omega}}(t(1-t))^{k-\alpha}\left|f^{(k)}(t)\right| \leq\|f\|_{\infty, \alpha, N},
$$

that on writing $k=j+l$ as above then

$$
\max _{t \in \bar{\Omega}}(t(1-t))^{l-(\alpha-j)}\left|\left(f^{(j)}(t)\right)^{(l)}\right| \leq\|f\|_{\infty, \alpha, N} \quad \text { for } l=0(1)(N-j),
$$

so that $f^{(j)} \in W_{\infty, \alpha-j}^{(N-j)}(\Omega)$ and $\left\|f^{(j)}\right\|_{\infty, \alpha-j, N-j} \leq\|f\|_{\infty, \alpha, N}$. This completes the proof of $(c)$.

Note in passing that in [4] the analysis was done in a space which, following the work done by Kress [7], I denoted by $K_{N}^{\alpha}$. In the notation of this paper, $K_{N}^{\alpha}$ is the space $W_{1, \alpha}^{(N)}(\Omega)$. We are now in a position to give some 
further properties of functions in the space $W_{p, \alpha}^{(N)}(\Omega)$. In particular, we shall say something about the continuity of these functions and their derivatives on $\Omega$ and $\bar{\Omega}$. In the statement of Theorem 4 , the space $L_{1}(\Omega)$ is that of integrable functions on $\Omega$ but first, a definition.

Definition 3 (a) $f$ is locally integrable on $\Omega$, we write $f \in L_{1, l o c}(\Omega)$, if $\int_{a}^{b}|f(t)| d t<\infty$ for all $a, b \in \Omega$ such that $0<a<b<1$.

(b) If $f$ is continuous on $\bar{\Omega}$ with, in addition, $f(0)=f(1)=0$ we shall write $f \in \stackrel{0}{C}(\bar{\Omega})$.

Theorem 4 Suppose $f \in W_{p, \alpha}^{(N)}(\Omega)$ where $1 \leq p \leq \infty$, and $\alpha \in \mathbb{R}$.

(a) If $1 \leq p<\infty, N \in \mathbb{N}$ and $n \in \mathbb{N}_{0}$ is such that $n<\alpha+1 / q \leq n+1 \leq N$ then

$$
f^{(j)} \in \begin{cases}\stackrel{0}{C}(\bar{\Omega}), & j=0(1)(n-1), \\ C(\Omega) \cap L_{1}(\Omega), & j=n, \\ C(\Omega), & j=(n+1)(1)(N-1), \\ L_{1, l o c}(\Omega), & j=N .\end{cases}
$$

(b) If $p=\infty, N \in \mathbb{N}_{0}$ and $n \in \mathbb{N}_{0}$ is such that $n<\alpha+1 \leq n+1$ then

$$
\left|f^{(j)}(t)\right| \leq\|f\|_{\infty, \alpha, N}(t(1-t))^{\alpha-j}
$$

for $j=0(1) N$ so that

$$
f^{(j)} \in \begin{cases}\stackrel{0}{C}(\bar{\Omega}), & j=0(1)(n-1), \\ C(\bar{\Omega}), & j=n \text { and } \alpha=n, \\ C(\Omega), & j=n \text { and } \alpha<n, \\ C(\Omega), & j=(n+1)(1) N .\end{cases}
$$




\section{Proof:}

(a) If $0<a<b<1$ then, by Hölder's inequality,

$$
\int_{a}^{b}\left|f^{(N)}(t)\right| d t \leq \begin{cases}\|f\|_{p, \alpha, N}\left(\int_{a}^{b}(t(1-t))^{q(\alpha-N)} d t\right)^{1 / q}, & 1<p<\infty, \\ \|f\|_{1, \alpha, N} \max _{t \in[a, b]}(t(1-t))^{\alpha-N}, & p=1 .\end{cases}
$$

Hence, by Definition $3, f^{(N)} \in L_{1, \text { loc }}(\Omega)$.

For $j \in\{0,1,2, \ldots, N-1\}$ let us first choose any $x, x+h$ in $\Omega$. Then

$$
\begin{aligned}
& \left|f^{(j)}(x+h)-f^{(j)}(x)\right| \\
& =\left|\int_{x}^{x+h} f^{(j+1)}(t) d t\right| \\
& =\left|\int_{x}^{x+h}(t(1-t))^{j+1-\alpha} f^{(j+1)}(t)(t(1-t))^{\alpha-j-1} d t\right| \\
& \leq \begin{cases}\|f\|_{p, \alpha, N}\left(\left|\int_{x}^{x+h}(t(1-t))^{q(\alpha-j-1)} d t\right|\right)^{1 / q}, & 1<p<\infty, \\
\|f\|_{1, \alpha, N} \max _{t \in\langle x, x+h\rangle}(t(1-t))^{\alpha-j-1}, & p=1,\end{cases}
\end{aligned}
$$

on using Hölder's inequality. Note that we write $t \in\langle x, x+h\rangle$ to mean that $x \leq t \leq x+h$ if $h>0$ and $x+h \leq t \leq x$ if $h<0$. For $1 \leq p<\infty$ we see that $\lim _{h \rightarrow 0} f^{(j)}(x+h)=f^{(j)}(x)$ and, since $x$ was chosen arbitrarily from $\Omega$, we have that $f^{(j)}$ is continuous on $\Omega$ for $j=0(1)(N-1)$. Suppose, however, that we limit $j$ to the set $\{0,1,2, \ldots, n-1\}$ then we may now assume that $x, x+h$ are points out of $\bar{\Omega}$. Since $q(\alpha-j-1)>-1$ then, when $1<p<\infty$, the integral exists even when we choose either $x$ or $x+h$ to be an end-point of $\bar{\Omega}$. Since, when $p=1, n<\alpha$ it follows that $\alpha-j-1>0$ for $j=0(1)(n-1)$ so that we shall have $f^{(j)} \in C(\bar{\Omega})$ for $j=0(1)(n-1)$ and $1 \leq p<\infty$. But we can say more. Since $f \in W_{p, \alpha}^{(N)}(\Omega)$ for $1 \leq p<\infty$ we have

$$
\int_{0}^{1}\left[(t(1-t))^{p(j-\alpha)}\left|f^{(j)}(t)\right|\right]^{p} d t<\infty
$$


for $j=0(1)(n-1)$. We have that $f^{(j)}$ is continuous on $\bar{\Omega}$ but since $n<\alpha+1 / q$, we have that $p(j-\alpha) \leq p(n-1-\alpha)<-1$. The only way that this integral can exist is if

$$
f^{(j)}(0)=f^{(j)}(1)=0 \quad \text { for } j=0(1)(n-1) .
$$

That is, $f^{(j)} \in \stackrel{0}{C}(\bar{\Omega})$ for $j=0(1)(n-1)$. If $\alpha+1 / q \leq n+1$ then we must have

$$
\int_{0}^{1} \frac{\left|f^{(n)}(t)\right|^{p}}{(t(1-t))^{p(\alpha-n)}} d t<\infty
$$

and although $p(\alpha-n) \leq 1$, given that $f^{(n)} \in C(\Omega)$ we can make no further conclusion about the values of $f^{(n)}$ at 0 and 1 . However, we shall have $f^{(n)} \in L_{1}(\Omega)$ since

$$
\begin{aligned}
\int_{0}^{1}\left|f^{(n)}(t)\right| d t & =\int_{0}^{1}(t(1-t))^{n-\alpha}\left|f^{(n)}(t)\right|(t(1-t))^{\alpha-n} d t \\
& \leq \begin{cases}\|f\|_{p, \alpha, N}\left(\int_{0}^{1}(t(1-t))^{q(\alpha-n)} d t\right)^{1 / q}, & 1<p<\infty, \\
\|f\|_{1, \alpha, N} \max _{t \in \bar{\Omega}}(t(1-t))^{\alpha-n}, & p=1 .\end{cases}
\end{aligned}
$$

Since $n<\alpha+1 / q$, the right hand side of (18) is finite for $1 \leq p<\infty$ which concludes the proof of (a).

(b) Consider now the case where $p=\infty$. From (2) it follows that

$$
(t(1-t))^{j-\alpha}\left|f^{(j)}(t)\right| \leq\|f\|_{\infty, \alpha, N}
$$

for $j=0(1) N$ and $t \in \bar{\Omega}$. Inequality (15) follows at once for every $t \in \Omega$ and $j=0(1) N$. Again, Definition 1(b) assumes that $f^{(j)} \in C(\Omega)$ for all $j=0(1) N$. Since we have assumed that $n<\alpha+1 \leq n+1$ it follows that $\alpha-j>0$ for $j=0(1)(n-1)$. Consequently $f^{(j)} \in \stackrel{0}{C}(\bar{\Omega})$ for $j=0(1)(n-1)$. In the particular case when $j=n=\alpha$, (15) implies that $\lim _{t \rightarrow 0}\left|f^{(n)}(t)\right|$ is finite, not necessarily zero. With a similar result at the end point 1 , it follows that $f^{(n)} \in C(\bar{\Omega})$ when $n=\alpha$. 
This completes the proof.

This theorem has established the qualitative nature of the functions and their derivatives given that they are in a weighted Sobolev space. In the special case when $p=\infty,(15)$ gives a bound on the function and its derivatives on $\Omega$. This raises the question as to whether similar results may be obtained in the general case when $1 \leq p<\infty$. This is the topic of the next theorem.

Theorem 5 Suppose $f \in W_{p, \alpha}^{(N)}(\Omega)$ where $1 \leq p \leq \infty, n \in \mathbb{N}_{0}$ and $N \in \mathbb{N}$ such that

$$
n<\alpha+1 / q \leq n+1 \leq N .
$$

Then for all $t \in \Omega$ and $j=0(1)(N-1)$

$$
\left|f^{(j)}(t)\right| \leq c\|f\|_{p, \alpha, N}(t(1-t))^{\alpha-1 / p-j},
$$

except when $\alpha+1 / q=n+1,1<p<\infty$ and $j=n$, in which case

$$
\left|f^{(n)}(t)\right| \leq c\|f\|_{p, \alpha, N} \ln ^{1 / q}[1 /(t(1-t))] .
$$

The constant $c$ is independent of $f$ and $t$.

Proof: Note that when $p=\infty$, (21) follows at once from (15) with $c=1$ and indeed in this case, the result is also true when $j=N$. We now restrict ourselves to the case when $1 \leq p<\infty$.

From Theorem 4 (a) since $f^{(j)}(0)=0$ for $j=0(1)(n-1)$ then

$$
f^{(j)}(t)=\int_{0}^{t} f^{(j+1)}(s) d s=\int_{0}^{t}(s(1-s))^{j+1-\alpha} f^{(j+1)}(s)(s(1-s))^{\alpha-j-1} d s .
$$

By Hölder's inequality we have

$$
\left|f^{(j)}(t)\right| \leq\|f\|_{p, \alpha, N} G_{j}(t)
$$


say, where the function

$$
G_{j}(t):= \begin{cases}\left(\int_{0}^{t}(s(1-s))^{q(\alpha-j-1)} d s\right)^{1 / q}, & 1<p<\infty \\ \max _{s \in[0, t]}(s(1-s))^{\alpha-j-1}, & p=1 .\end{cases}
$$

If $1<p<\infty$ then, on writing $s=t u$, we have

$$
G_{j}(t)=t^{\alpha-j-1+1 / q}\left(\int_{0}^{1}(u(1-t u))^{q(\alpha-j-1)} d u\right)^{1 / q} .
$$

Assume first that $0 \leq t \leq 1 / 2$. Since $1 / 2 \leq 1-t u \leq 1$ for all $0 \leq u \leq 1$ and since $q(\alpha-j-1)>-1$ for $j=0(1)(n-1)$ we have from (26) that

$$
G_{j}(t) \leq c \cdot t^{\alpha-j-1-1 / q}
$$

where $c \neq c(t)$. (Note that throughout we shall take $c$ to denote a generic constant whose value may change from line to line. The notation $c \neq c(t)$ means that $c$ is independent of $t$.) From (3), (24), (25) and (27), we have

$$
\left|f^{(j)}(t)\right| \leq c\|f\|_{p, \alpha, N} t^{\alpha-j-1 / p}
$$

for $0 \leq t \leq 1 / 2$ and $j=0(1)(n-1)$. We argue similarly when $t \in[1 / 2,1]$. Since $f^{(j)}(1)=0$ for $j=0(1)(n-1)$, from

$$
f^{(j)}(t)=\int_{1}^{t} f^{(j+1)}(s) d s
$$

we find that

$$
\left|f^{(j)}(t)\right| \leq c\|f\|_{p, \alpha, N}(1-t)^{\alpha-j-1 / p}
$$

for $1 / 2 \leq t \leq 1$. Since for $j=0(1)(n-1)$ we have $\alpha-j-1 / p>0$ we may combine (28) and (29) to give

$$
\left|f^{(j)}(t)\right| \leq c\|f\|_{p, \alpha, N}(t(1-t))^{\alpha-j-1 / p}
$$


for all $t \in \bar{\Omega}$. When $p=1$, we have at once from (25) that

$$
G_{j}(t) \leq c \cdot(t(1-t))^{\alpha-j-1}, \quad t \in \bar{\Omega},
$$

since $\alpha-j-1>0$ for $j=0(1)(n-1)$ and $1 / q=0$ in this case. Again (24), (25) and (31) combine to give (21) for $j=0(1)(n-1)$ when $p=1$.

Suppose now that $j \in\{n, n+1, \ldots, N-1\}$. From Theorem 4(a) we know that $f^{(j)}$ is continuous on $\Omega$. In particular, choose any point $a \in \Omega$ and suppose $t$ is such that $0<a \leq t<1$. Then

$f^{(j)}(t)-f^{(j)}(a)=\int_{a}^{t} f^{(j+1)}(s) d s=\int_{a}^{t}\left(s(1-s)^{j+1-\alpha} f^{(j+1)}(s)(s(1-s))^{\alpha-j-1} d s\right.$.

By Hölder's inequality we have

$$
\left|f^{(j)}(t)-f^{(j)}(a)\right| \leq\|f\|_{p . \alpha, N} H_{j}(t)
$$

say, where the function

$$
H_{j}(t):= \begin{cases}\left(\int_{a}^{t}(s(1-s))^{(\alpha-j-1) q} d s\right)^{1 / q}, & 1<p<\infty, \\ \max _{a \leq s \leq t}(s(1-s))^{\alpha-j-1}, & p=1 .\end{cases}
$$

Suppose that $1<p<\infty$. Since $(\alpha-j-1) q \leq-1$ for $j=n(1)(N-1)$ we have, by Hölder's inequality for sums, that

$$
(s(1-s))^{(\alpha-j-1) q}=\left(\frac{1}{s}+\frac{1}{1-s}\right)^{(j+1-\alpha) q} \leq c\left(\frac{1}{s^{(j+1-\alpha) q}}+\frac{1}{(1-s)^{(j+1-\alpha) q}}\right),
$$

for some $c$ independent of $s$, so that

$$
H_{j}(t) \leq c\left(\int_{a}^{t} \frac{1}{s^{(j+1-\alpha) q}}+\frac{1}{(1-s)^{(j+1-\alpha) q}} d s\right)^{1 / q} .
$$


On assuming that $(j+1-\alpha) q>1$ we find from (34) that

$$
\begin{aligned}
H_{j}^{q}(t) \leq & \frac{c}{(j+1-\alpha) q-1}\left(\frac{1}{(1-t)^{(j+1-\alpha) q-1}}-\frac{1}{(1-a)^{(j+1-\alpha) q-1}}\right. \\
& \left.-\frac{1}{t^{(j+1-\alpha) q-1}}+\frac{1}{a^{(j+1-\alpha) q-1}}\right) .
\end{aligned}
$$

Since $0<a \leq t<1$ we find that

$$
\begin{aligned}
H_{j}^{q}(t) & \leq \frac{c}{(j+1-\alpha) q-1}\left(\frac{1}{(1-t)^{(j+1-\alpha) q-1}}+\frac{1}{a^{(j+1-\alpha) q-1}}\right) \\
& \leq \frac{c}{(a(1-t))^{(j+1-\alpha) q-1}} .
\end{aligned}
$$

From (32) and (35) we have

$$
\left|f^{(j)}(t)\right| \leq\left|f^{(j)}(a)\right|+\frac{c\|f\|_{p, \alpha, N}}{(a(1-t))^{j+1-\alpha-1 / q}}
$$

for $0<a \leq t<1$. We may proceed analogously when $0<t \leq a<1$ to give in this case that

$$
\left|f^{(j)}(t)\right| \leq\left|f^{(j)}(a)\right|+\frac{c\|f\|_{p, \alpha, N}}{(t(1-a))^{j+1-\alpha-1 / q}} .
$$

Combining (36) and (37) gives

$$
\left|f^{(j)}(t)\right| \leq\left|f^{(j)}(a)\right|+\frac{c\|f\|_{p, \alpha, N}}{(t(1-t))^{j+1-\alpha-1 / q}(a(1-a))^{j+1-\alpha-1 / q}},
$$

for any $a \in \Omega$ and $t \in \Omega$. The constant $c$ is independent of $a, t$ and the function $f$. We shall now fix $t$ in (38) and consider $a$ as a variable over $\Omega$. Recall from Theorem 2(b) that if $f \in W_{p, \alpha}^{(N)}(\Omega)$ then also $f \in W_{p, \beta}^{(N)}(\Omega)$ for any $\beta<\alpha$ with $\|f\|_{p, \beta, N} \leq c\|f\|_{p, \alpha, N}$. In (38), by Hölder's inequality for sums, we have

$$
\left|f^{(j)}(t)\right|^{p} \leq c \cdot\left(\left|f^{(j)}(a)\right|^{p}+\frac{\|f\|_{p, \alpha, N}^{p}}{(t(1-t))^{p(j+1-\alpha-1 / q)}(a(1-a))^{p(j+1-\alpha-1 / q)}}\right) .
$$


On multiplying each side of (39) by $(a(1-a))^{p(j-\beta)}$ and integrating over $(0,1)$ with respect to $a$ we find

$$
\begin{aligned}
& \left|f^{(j)}(t)\right|^{p} \cdot \int_{0}^{1}(a(1-a))^{p(j-\beta)} d a \\
& \leq c\left(\|f\|_{p, \beta, N}^{p}+\frac{\|f\|_{p, \alpha, N}^{p}}{(t(1-t))^{p(j+1-\alpha-1 / q)}} \int_{0}^{1}(a(1-a))^{p(\alpha-\beta)-1} d a\right) .
\end{aligned}
$$

The integral on the left hand side of (40) exists since for $j=n(1)(N-1)$ we have $p(j-\beta)>-1$. Again the integral on the right hand side of (40) exists since $\alpha>\beta$. Consequently from (40) we have

$$
\left|f^{(j)}(t)\right|^{p} \leq c\left(\|f\|_{p, \beta, N}^{p}+\frac{\|f\|_{p, \alpha, N}^{p}}{(t(1-t))^{p(j+1-\alpha-1 / q)}}\right)
$$

for some $c$ independent of $t$ and $f$. Finally, since $\|f\|_{p, \beta, N} \leq c\|f\|_{p, \alpha, N}$ we find from (41) that

$$
\left|f^{(j)}(t)\right| \leq \frac{c\|f\|_{p, \alpha, N}}{(t(1-t))^{j-\alpha+1 / p}}
$$

as required.

In the particular case when $n+1=\alpha+1 / q$ so that $q(\alpha-n-1)=-1$, we have from (33) with $1<p<\infty$ that

$$
H_{n}^{q}(t)=\int_{a}^{t}\left(\frac{1}{s}+\frac{1}{1-s}\right) d s=\ln \left(\frac{t(1-a)}{a(1-t)}\right) .
$$

Since $0<a \leq t<1$ we have

$$
H_{n}(t) \leq \ln ^{1 / q}\left(\frac{1}{a(1-t)}\right)
$$

Similarly, for $0<t \leq a<1$, we find

$$
H_{n}(t) \leq \ln ^{1 / q}\left(\frac{1}{t(1-a)}\right)
$$


We may combine (44) and (45) so that for $0<a<1$ and $0<t<1$ we have

$$
H_{n}(t) \leq \ln ^{1 / q}\left(\frac{1}{t(1-t) a(1-a)}\right) .
$$

Since for $a, t \in \Omega$, we have both $1 /(t(1-t))$ and $1 /(a(1-a))$ are $\geq 4$ it follows that

$$
\ln \left(\frac{1}{t(1-t) a(1-a)}\right) \leq \frac{1}{\ln 2} \ln \left(\frac{1}{t(1-t)}\right) \ln \left(\frac{1}{a(1-a)}\right) .
$$

From (32), (46) and (47) we have

$$
\left|f^{(n)}(t)\right| \leq\left|f^{(n)}(a)\right|+c\|f\|_{p, \alpha, N} \ln ^{1 / q}\left(\frac{1}{t(1-t)}\right) \ln ^{1 / q}\left(\frac{1}{a(1-a)}\right),
$$

so that

$$
\left|f^{(n)}(t)\right|^{p} \leq c\left[\left|f^{(n)}(a)\right|^{p}+c\|f\|_{p, \alpha, N}^{p} \ln ^{p / q}\left(\frac{1}{t(1-t)}\right) \ln ^{p / q}\left(\frac{1}{a(1-a)}\right)\right],
$$

for some positive constant $c$ independent of $f, a$ and $t$. Arguing as before, if we multiply each side of $(49)$ by $(a(1-a))^{p(n-\beta)}$ where $\beta<\alpha$ and integrate with respect to $a$ over $(0,1)$ we find

$$
\begin{aligned}
& \left|f^{(n)}(t)\right|^{p} \cdot \int_{0}^{1}(a(1-a))^{p(n-\beta)} d a \\
& \leq c\left\{\|f\|_{p, \beta, N}^{p}+\|f\|_{p, \alpha, N}^{p} \ln ^{p / q}\left(\frac{1}{t(1-t)}\right) \times\right. \\
& \left.\quad \times \int_{0}^{1}(a(1-a))^{p(n-\beta)} \ln ^{p / q}\left(\frac{1}{a(1-a)}\right) d a\right\} .
\end{aligned}
$$

Since both integrals exist and since $\|f\|_{p, \beta, N} \leq c\|f\|_{p, \alpha, N}$ it follows from (50) that

$$
\left|f^{(n)}(t)\right| \leq c\|f\|_{p, \alpha, N} \ln ^{1 / q}\left(\frac{1}{t(1-t)}\right)
$$


for all $t \in \Omega$, which is (22).

It finally remains to consider the case when $p=1$. For $j=0(1)(n-1)$ we have that $\alpha-j-1>0$ since, from(20), when $p=1$ we have $1 / q=0$ so that $n<\alpha$. Consequently recalling $(25)$ gives $G_{j}(t)=(t(1-t))^{\alpha-j-1}$ and from (24) we see that

$$
\left|f^{(j)}(t)\right| \leq\|f\|_{1, \alpha, N}(t(1-t))^{\alpha-j-1}
$$

which is (21) with $p=1,1 / q=0$ and $c=1$.

For $j=n(1)(N-1)$ and $n<\alpha \leq n+1$ from (33) we have, since $j+1 \geq \alpha$, that

$$
H_{j}(t) \leq \max \left\{\frac{1}{(a(1-a))^{j+1-\alpha}}, \frac{1}{(t(1-t))^{j+1-\alpha}}\right\} .
$$

Consequently (32) gives in this case that

$$
\left|f^{(j)}(t)\right| \leq\left|f^{(j)}(a)\right|+\|f\|_{1, \alpha, N} \max \left\{\frac{1}{(a(1-a))^{j+1-\alpha}}, \frac{1}{(t(1-t))^{j+1-\alpha}}\right\} .
$$

We shall argue as before by choosing $\beta<\alpha$ then, since $f \in W_{1, \alpha}^{(N)}(\Omega)$, we know that $f \in W_{1, \beta}^{(N)}(\Omega)$ also, with $\|f\|_{1, \beta, N} \leq\|f\|_{1, \alpha, N}$. On multiplying (54) by $(a(1-a))^{j-\beta}$ and integrating with respect to $a$ over $(0,1)$ we find

$$
\begin{aligned}
\left|f^{(j)}(t)\right| \cdot \int_{0}^{1}(a(1-a))^{j-\beta} d a & \\
\leq\|f\|_{1, \beta, N}+\|f\|_{1, \alpha, N} \max \{ & \left\{\int_{0}^{1}(a(1-a))^{\alpha-\beta-1} d a,\right. \\
& \left.\frac{1}{(t(1-t))^{j+1-\alpha}} \int_{0}^{1}(a(1-a))^{j-\beta} d a\right\} .
\end{aligned}
$$

Obviously all the integrals exist so that we find

$$
\left|f^{(j)}(t)\right| \leq c\|f\|_{1, \alpha, N}(t(1-t))^{\alpha-j-1}
$$


for $j=n(1)(N-1)$ given that $n<\alpha \leq n+1$ and $p=1$. This is (21) and completes the proof.

From Theorem 4(a) we see that given $f \in W_{p, \alpha}^{(N)}(\Omega)$ for $1 \leq p<\infty$ and with $N \geq n+1$ then, for $j=0(1)(N-1), f^{(j)}$ will be continuous on $\Omega$. From Theorem 5 we have the behaviour of $f^{(j)}$ near the end points of $\Omega$ for $j=0(1)(N-1)$. These results come together in the following theorem which will be of some importance in the subsequent analysis.

Theorem 6 (a) Suppose, for $1<p<\infty$, that $n<\alpha+1 / q<n+1 \leq N$ or, for $p=1$, that $n<\alpha \leq n+1 \leq N$ where $\alpha \in \mathbb{R}, n \in \mathbb{N}_{0}$ and $N \in \mathbb{N}$. Then if $f \in W_{p, \alpha}^{(N)}(\Omega)$ we also have that $f \in W_{\infty, \alpha-1 / p}^{(N-1)}(\Omega)$ with

$$
\|f\|_{\infty, \alpha-1 / p, N-1} \leq c\|f\|_{p, \alpha, N}
$$

for some constant $c$ independent of $f$.

(b) Suppose, for $\alpha \in \mathbb{R}, f \in W_{\infty, \alpha}^{(N)}(\Omega)$ with $n<\alpha+1 \leq n+1$ where $n, N \in \mathbb{N}_{0}$. Then for any $1 \leq p<\infty$ we have $f \in W_{p, \gamma}^{(N)}(\Omega)$ where $\gamma<\alpha+1 / p$. Furthermore,

$$
\|f\|_{p, \gamma, N} \leq c\|f\|_{\infty, \alpha, N}
$$

for some constant $c$ independent of $f$.

\section{Proof:}

(a) In each case we need to consider, for $j=0(1)(N-1)$,

$$
I_{j}:=\max _{t \in \bar{\Omega}}(t(1-t))^{j-(\alpha-1 / p)}\left|f^{(j)}(t)\right| .
$$

From (21) we have for $1 \leq p<\infty$ that

$$
\left|f^{(j)}(t)\right| \leq c\|f\|_{p, \alpha, N}(t(1-t))^{\alpha-1 / p-j},
$$


for $j=0(1)(N-1)$. Combining (59) and (60) gives

$$
I_{j} \leq c\|f\|_{p, \alpha, N}<\infty
$$

for $j=0(1)(N-1)$, so that $f \in W_{\infty, \alpha-1 / p}^{(N-1)}(\Omega)$ and (57) follows at once.

(b) Since $f \in W_{\infty, \alpha}^{(N)}(\Omega)$ we have, from Definition $1(\mathrm{~b})$ that

$$
\left|f^{(j)}(t)\right| \leq\|f\|_{\infty, \alpha, N}(t(1-t))^{\alpha-j}
$$

for $j=0(1) N$. Consider $I_{j}$, for $j=0(1) N$, where we now define

$$
I_{j}:=\int_{0}^{1}\left[(t(1-t))^{(j-\alpha)}\left|f^{(j)}(t)\right|\right]^{p} d t .
$$

From (62) and (63) we have

$$
I_{j} \leq\|f\|_{\infty, \alpha, N} \int_{0}^{1}(t(1-t))^{p(\alpha-\gamma)} d t \leq c\|f\|_{\infty, \alpha, N}
$$

provided $\gamma$ is chosen so that $p(\alpha-\gamma)>-1$, which is so. Since $I_{j}<\infty$ for $j=0(1) N$ it follows that $f \in W_{p, \gamma}^{(N)}(\Omega)$ and (58) follows at once.

There is a further consequence of Theorem 5 which will be useful when, in Section 4, we come to consider estimates of the truncation error in the Euler-Maclaurin formula. We shall define the function $\rho$ by

$$
\rho(t):=t(1-t) \text {. }
$$

Theorem 7 Suppose $f \in W_{p, \alpha}^{(N)}(\Omega)$ where $1 \leq p \leq \infty, n \in \mathbb{N}_{0}$ is such that $n<\alpha+1 / q \leq n+1$ and $N \in \mathbb{N}$ with $N \geq n+1$. Then
(a) $\rho f^{(n)} \in \stackrel{0}{C}(\bar{\Omega})$, and
(b) $\rho f^{(n+1)} \in L_{1}(\Omega)$. 


\section{Proof:}

(a) By the preceding theorem we have that for all $1 \leq p \leq \infty$, the function $f \in W_{\infty, \alpha-1 / p}^{(N-1)}(\Omega)$ so that

$$
(t(1-t))^{n-(\alpha-1 / p)}\left|f^{(n)}(t)\right| \leq\|f\|_{\infty, \alpha-1 / p, N-1} .
$$

Thus

$$
\rho(t)\left|f^{(n)}(t)\right| \leq c\|f\|_{p, \alpha, N}(t(1-t))^{\alpha+1 / q-n}
$$

and the right-hand side tends to zero as parameter $t \rightarrow 0$ and 1 . That is, $\rho f^{(n)} \in \stackrel{0}{C}(\bar{\Omega})$ as required.

(b) Consider

$$
\int_{0}^{1}(t(1-t))\left|f^{(n+1)}(t)\right| d t=\int_{0}^{1}(t(1-t))^{n+1-\alpha}\left|f^{(n+1)}(t)\right|(t(1-t))^{\alpha-n} d t .
$$

By Hölder's inequality we have

$$
\begin{aligned}
& \int_{0}^{1} t(1-t)\left|f^{(n+1)}(t)\right| d t \\
& \leq \begin{cases}\|f\|_{p, \alpha, N}\left(\int_{0}^{1}(t(1-t))^{q(\alpha-n)} d t\right)^{1 / q}, & 1<p<\infty, \\
\|f\|_{1, \alpha, N} \max _{t \in \bar{\Omega}}(t(1-t))^{\alpha-n}, & p=1 .\end{cases}
\end{aligned}
$$

Since $n<\alpha+1 / q$ we have $q(\alpha-n) \geq-1$ so that the integral on the right of (66) exists. Again, when $p=1, \alpha-n>0$ so that $\max _{t \in \bar{\Omega}}(t(1-t))^{\alpha-n}=(1 / 4)^{\alpha-n}$. This establishes (b) and the theorem is proved.

As a final result in this section we need to determine the weighted Sobolev space in which the product of two functions is to be found. But first, it is an 
easy matter to show that if $p$ and $q$ are conjugate numbers, recall (3), and if $r$ is such that $q \leq r \leq \infty$ then $s$, defined by

$$
\frac{1}{s}:=\frac{1}{p}+\frac{1}{r}
$$

is such that $1 \leq s \leq \infty$.

Theorem 8 Suppose $p$ and $q$ are conjugate numbers with $1 \leq p, q \leq \infty$ and $r$ is such that $q \leq r \leq \infty$. If $f \in W_{p, \alpha}^{\left(N_{1}\right)}(\Omega)$ and $g \in W_{r, \beta}^{\left(\overline{N_{2}}\right)}(\Omega)$ for some $\alpha, \beta \in \mathbb{R}$ and $N_{1}, N_{2} \in \mathbb{N}_{0}$ then $f g \in W_{s, \alpha+\beta}^{(N)}(\Omega)$ where $1 / s=1 / p+1 / r$ and $N=\min \left\{N_{1}, N_{2}\right\}$. Furthermore,

$$
\|f g\|_{s, \alpha+\beta, N} \leq c\|f\|_{p, \alpha, N_{1}}\|g\|_{r, \beta, N_{2}}
$$

where $c$ is a positive constant such that $c \neq c(f, g)$.

Proof: Since $N=\min \left\{N_{1}, N_{2}\right\}$ it follows at once from Definition 1 that $f \in W_{p, \alpha}^{(N)}(\Omega)$ and $g \in W_{r, \beta}^{(N)}(\Omega)$ with

$$
\|f\|_{p, \alpha, N} \leq\|f\|_{p, \alpha, N_{1}} \quad \text { and } \quad\|g\|_{r, \beta, N} \leq\|g\|_{r, \beta, N_{2}} .
$$

Suppose first that $s=\infty$. This can only occur if $p=r=\infty$. For $j=0(1) N$, consider

$$
I_{j}(\infty):=\max _{t \in \bar{\Omega}}(t(1-t))^{j-(\alpha+\beta)}\left|(f g)^{(j)}(t)\right| .
$$

By Leibnitz's theorem we have

$$
(f g)^{(j)}(t)=\sum_{k=0}^{j}\left(\begin{array}{l}
j \\
k
\end{array}\right) f^{(k)}(t) g^{(j-k)}(t) .
$$


From (70) and (71) we have

$$
I_{j}(\infty) \leq \sum_{k=0}^{j}\left(\begin{array}{l}
j \\
k
\end{array}\right)\left[\max _{t \in \bar{\Omega}}(t(1-t))^{k-\alpha}\left|f^{(k)}(t)\right|\right]\left[\max _{t \in \bar{\Omega}}(t(1-t))^{j-k-\beta}\left|g^{(j-k)}(t)\right|\right]
$$

It follows at once that for $j=0(1) N$

$$
I_{j}(\infty) \leq 2^{j}\|f\|_{\infty, \alpha, N_{1}}\|g\|_{\infty, \alpha, N_{2}}
$$

since $\sum_{k=0}^{j}\left(\begin{array}{l}j \\ k\end{array}\right)=2^{j}$. Consequently $f g \in W_{\infty, \alpha+\beta}^{(N)}(\Omega)$ and (68) is satisfied with $c=2^{N}$.

Suppose now that $1 \leq s<\infty$ and consider

$$
I_{j}(s):=\int_{0}^{1}\left[(t(1-t))^{j-(\alpha+\beta)}\left|(f g)^{(j)}(t)\right|\right]^{s} d t,
$$

for $j=0(1) N$. Again by Leibnitz's theorem, see(71), we have

$$
I_{j}(s)=\int_{0}^{1}\left|\sum_{k=0}^{j}\left(\begin{array}{l}
j \\
k
\end{array}\right)(t(1-t))^{k-\alpha} f^{(k)}(t) \cdot(t(1-t))^{j-k-\beta} g^{(j-k)}(t)\right|^{s} d t .
$$

From the triangle inequality when $s=1$ and Hölder's inequality for sums when $1<s<\infty$ we have

$$
\left|\sum_{k=0}^{j} a_{k}\right|^{s} \leq(j+1)^{s-1} \sum_{k=0}^{j}\left|a_{k}\right|^{s}, \quad 1 \leq s \leq \infty .
$$

From (74) and (75) we have

$$
\begin{aligned}
I_{j}(s) \leq(j+1)^{s-1} \sum_{k=0}^{j}\left(\begin{array}{l}
j \\
k
\end{array}\right)^{s} \int_{0}^{1} & \left((t(1-t))^{k-\alpha}\left|f^{(k)}(t)\right|\right)^{s} \times \\
\times & \left((t(1-t))^{j-k-\beta}\left|g^{(j-k)}(t)\right|\right)^{s} d t
\end{aligned}
$$


for $j=0(1) N$. If $p=\infty$ then $s=r$ and since in this case

$$
\|f\|_{\infty, \alpha, N_{1}}=\max _{k=0(1) N_{1}} \max _{t \in \bar{\Omega}}(t(1-t))^{k-\alpha}\left|f^{(k)}(t)\right|
$$

we see from (76) that for $j=0(1) N$

$$
I_{j}(s) \leq(j+1)^{s-1}\|f\|_{\infty, \alpha, N_{1}}^{s}\|g\|_{r, \beta, N_{2}}^{s} \sum_{k=0}^{j}\left(\begin{array}{l}
j \\
k
\end{array}\right)^{s} .
$$

Consequently in this case $f g \in W_{s, \alpha+\beta}^{(N)}(\Omega)$ with

$$
\|f g\|_{s, \alpha+\beta, N} \leq c\|f\|_{\infty, \alpha, N_{1}}\|g\|_{r, \beta, N_{2}}
$$

where $c:=(j+1)^{1-1 / s}\left[\sum_{k=0}^{j}\left(\begin{array}{l}j \\ k\end{array}\right)^{s}\right]^{1 / s}$ is independent of both $f$ and $g$.

Arguing similarly if $r=\infty$ so that $s=p$ we would find

$$
\|f g\|_{s, \alpha+\beta, N} \leq c\|f\|_{p, \alpha, N_{1}}\|g\|_{\infty, \beta, N_{2}} .
$$

Thus it remains to consider $I_{j}(s)$, see inequality (76), when $1<s<\infty$ and $1 \leq p, r<\infty$. Under these circumstances we see from (67) that $p / s>1$ so that applying Hölder's inequality to the integral on the right of (76) we find

$$
\begin{aligned}
I_{j}(s) \leq(j+1)^{s-1} \sum_{k=0}^{j}\left(\begin{array}{l}
j \\
k
\end{array}\right)^{s} & {\left[\int_{0}^{1}\left[(t(1-t))^{k-\alpha}\left|f^{(k)}(t)\right|\right]^{p} d t\right]^{s / p} \times } \\
& \times\left[\int_{0}^{1}\left[(t(1-t))^{j-k-\beta}\left|g^{(k-j)}(t)\right|\right]^{r}\right]^{s / r} .
\end{aligned}
$$

Consequently

$$
I_{j}(s) \leq(j+1)^{s-1}\|f\|_{p, \alpha, N_{1}}^{s}\|g\|_{r, \beta, N_{2}}^{s} \sum_{k=0}^{j}\left(\begin{array}{l}
j \\
k
\end{array}\right)^{s}
$$

for $j=0(1) N$. Again we find that $f g \in W_{s, \alpha+\beta}^{(N)}(\Omega)$ and that (68) is satisfied with $c=(j+1)^{1-1 / s}\left[\sum_{k=0}^{j}\left(\begin{array}{l}j \\ k\end{array}\right)^{s}\right]^{1 / s}$. This completes the proof of the theorem. 


\section{Sigmoidal transformations}

Sigmoidal transformations are useful when the Euler-Maclaurin formula is used to find approximate values of the integral $\int_{0}^{1} f(x) d x$. Essentially we make a change of variable $x=\gamma_{r}(t)$ say, where $r>1$, and use the EulerMaclaurin sum on $\int_{0}^{1} g_{r}(t) d t$ where the function

$$
g_{r}(t):=f\left(\gamma_{r}(t)\right) \gamma_{r}^{\prime}(t), \quad t \in \Omega, \quad r>1 .
$$

The function $\gamma_{r}$ maps $\bar{\Omega}$ onto itself and is chosen so that $\gamma_{r}(t)=\mathcal{O}\left(t^{r}\right)$ as $t \rightarrow 0$, whereas near $t=1, \gamma_{r}(t)=1+\mathcal{O}\left((1-t)^{r}\right)$. There are many such transformations to be found in the literature; a survey has been given by the author in [5]. An archetypal transformation is

$$
\gamma_{r}(t)=\frac{t^{r}}{t^{r}+(1-t)^{r}} .
$$

Since the graph of this function is in the shape of an elongated "S", these transformations are called sigmoidal. This leads us to the following definition of a generic sigmoidal transformation of order $r$.

Definition 9 A sigmoidal transformation $\gamma_{r}$ of order $r>1$ possesses the following properties:

(a) $\gamma_{r} \in C^{1}(\bar{\Omega}) \cap C^{\infty}(\Omega)$ with $\gamma_{r}(0)=0$,

(b) $\gamma_{r}(t)+\gamma_{r}(1-t)=1$ for all $t \in \bar{\Omega}$,

(c) $\gamma_{r}$ is strictly increasing on $\bar{\Omega}$,

(d) $\gamma_{r}^{\prime}$ is strictly increasing on $[0,1 / 2]$ with $\gamma_{r}^{\prime}(0)=0$,

(e) $\gamma_{r}^{(j)}(t)=\mathcal{O}\left(t^{r-j}\right)$ near $t=0$, for all $j \in \mathbb{N}_{0}$ when $r \notin \mathbb{N}$ and for $j=0(1) r$ if $\mathbb{N} \ni r \geq 2$. 
As an immediate consequence of Definition 9, note, from the discussions of Section 2, that $\gamma_{r} \in W_{p, \alpha}^{(N)}(\Omega)$ for any $N \in \mathbb{N}_{0}$ where $\alpha+1 / q<1$ if $1 \leq p<\infty$ and $\alpha \leq 0$ if $p=\infty$. Again, for the function $\gamma_{r}^{\prime}$ it can be verified that $\gamma_{r}^{\prime} \in W_{p, \gamma}^{(N)}(\Omega)$ for any $N \in \mathbb{N}_{0}$ where $\gamma+1 / q<r$ if $1 \leq p<\infty$ and $\gamma+1 \leq r$ if $p=\infty$.

Before considering the Euler-Maclaurin formula, the fundamental question we shall now address is that given $f \in W_{p, \alpha}^{(N)}(\Omega)$ for $1 \leq p \leq \infty$ and $N$ "sufficiently large", in what space is the function $g_{r}$, as defined in (81), to be found? The answer is in the following theorem.

Theorem 10 Suppose $f \in W_{p, \alpha}^{(N)}(\Omega)$ where $1 \leq p \leq \infty$ and $N \in \mathbb{N}_{0}$ is sufficiently large. If $g_{r}:=\left(f \circ \gamma_{r}\right) \gamma_{r}^{\prime}$ then $g_{r} \in W_{p, \alpha r+(r-1) / q}^{(N)}(\Omega)$ and

$$
\left\|g_{r}\right\|_{p, \alpha r+(r-1) / q, N} \leq c\|f\|_{p, \alpha, N}
$$

where $c$ is a positive constant independent of $f$.

Proof: Choose $j \in\{0,1, \ldots, N\}$. From (81), by Leibnitz' theorem, we have

$$
g_{r}^{(j)}(t)=\sum_{k=0}^{j}\left(\begin{array}{l}
j \\
k
\end{array}\right)\left(\gamma_{r}^{\prime}(t)\right)^{(j-k)}\left(f\left(\gamma_{r}(t)\right)\right)^{(k)} .
$$

Let us rewrite this as

$$
g_{r}^{(j)}(t)=\sum_{k=0}^{j} u_{k, j}(t) f^{(k)}\left(\gamma_{r}(t)\right),
$$

say. On differentiating (85) once we find that the functions $u_{k, j}$ satisfy

$$
\left\{\begin{array}{l}
u_{0, j+1}(t)=u_{0, j}^{\prime}(t) \\
u_{s, j+1}(t)=u_{s-1, j}(t) \gamma_{r}^{\prime}(t)+u_{s, j}^{\prime}(t), \quad s=1(1) j, \\
u_{j+1, j+1}(t)=u_{j, j}(t) \gamma_{r}^{\prime}(t) .
\end{array}\right.
$$


In particular we see that since, from $(81), u_{0,0}(t)=\gamma_{r}^{\prime}(t)$ then

$$
u_{0, j}(t)=\gamma_{r}^{(j+1)}(t) \quad \text { and } \quad u_{j, j}(t)=\left(\gamma_{r}^{\prime}(t)\right)^{j+1} .
$$

In order to identify the space in which $g_{r}$ is to be found let us consider its behaviour near the end points of $\Omega$. In particular, near $t=0$, we have from (87) and Definition 9 that

$$
u_{0, j}(t)=\mathcal{O}\left(t^{r-j-1}\right) \quad \text { and } \quad u_{j, j}(t)=\mathcal{O}\left(t^{(r-1)(j+1)}\right) .
$$

What about the behaviour of $u_{s, j}(t)$ near $t=0$ for $s=1(1)(j-1)$ ? From (86) if we conjecture that near $t=0$

$$
u_{s, j}(t)=\mathcal{O}\left(t^{r-1+r s-j}\right), \quad \text { for } \quad s=0(1) j,
$$

then it is readily verified that (86) and (88) are satisfied. Similarly, near $t=1$ we shall have

$$
u_{s, j}(t)=O\left((1-t)^{r-1+r s-j}\right), \quad s=0(1) j .
$$

Consequently we may write

$$
u_{s, j}(t)=(t(1-t))^{r-1+r s-j} U_{s, j}(t)
$$

say, for $s=0(1) j$ and $j=0(1) N$ where the functions $U_{s, j}$ are continuous on $\bar{\Omega}$ and non-vanishing at the end-points. In the same spirit and bearing in mind Definition 9 we may write

$$
\begin{aligned}
& \gamma_{r}(t)\left(1-\gamma_{r}(t)\right)=(t(1-t))^{r} \Gamma_{r, 0}(t), \\
& \gamma_{r}^{\prime}(t)=(t(1-t))^{r-1} \Gamma_{r, 1}(t)
\end{aligned}
$$

say, where $\Gamma_{r, 0}$ and $\Gamma_{r, 1}$ are continuous and strictly positive on $\bar{\Omega}$.

Suppose now that $1 \leq p<\infty$ and, for a given $j \in\{0,1,2, \ldots, N\}$, consider

$$
I_{j}(p):=\int_{0}^{1}\left[(t(1-t))^{j-\alpha r-(r-1) / q}\left|g_{r}^{(j)}(t)\right|\right]^{p} d t .
$$


From (57) and (85) we have

$$
\left|g_{r}^{(j)}(t)\right|^{p} \leq c \sum_{k=0}^{j}\left|u_{k, j}(t)\right|^{p}\left|f^{(k)}\left(\gamma_{r}(t)\right)\right|^{p}
$$

for some constant $c$ depending only on $j$ and $p$. Consequently,

$$
I_{j}(p) \leq c \sum_{k=0}^{j} \int_{0}^{1}\left[(t(1-t))^{j-\alpha r-r+1}\left|u_{k, j}(t)\right|\left|f^{(k)}\left(\gamma_{r}(t)\right)\right|\right]^{p}[t(1-t)]^{r-1} d t .
$$

From (91) and (93) we have

$$
I_{j}(p) \leq c \sum_{k=0}^{j} \int_{0}^{1}\left[(t(1-t))^{r(k-\alpha)}\left|U_{k, j}(t)\right|\left|f^{(k)}\left(\gamma_{r}(t)\right)\right|\right]^{p} \frac{\gamma_{r}^{\prime}(t)}{\Gamma_{r, 1}(t)} d t .
$$

From (92) and since $U_{k, j}, 1 / \Gamma_{r, 0}$ and $1 / \Gamma_{r, 1}$ are continuous on $\bar{\Omega}$ we find

$$
I_{j}(p) \leq c \sum_{k=0}^{j} \int_{0}^{1}\left[\left(\gamma_{r}(t)\left(1-\gamma_{r}(t)\right)\right)^{k-\alpha}\left|f^{(k)}\left(\gamma_{r}(t)\right)\right|\right]^{p} \gamma_{r}^{\prime}(t) d t
$$

where again $c$ is independent of $f$. On writing $x=\gamma_{r}(t)$ we find that

$$
I_{j}(p) \leq c \sum_{k=0}^{j} \int_{0}^{1}\left[(x(1-x))^{k-\alpha}\left|f^{(k)}(x)\right|\right]^{p} d x \leq c\|f\|_{p, \alpha, N}^{p}
$$

by (1). As this is true for $j=0(1) N$ we have at once that $g_{r} \in W_{p, \alpha r+(r-1) / q}^{N}(\Omega)$ for $1 \leq p<\infty$ and (83) is satisfied.

It remains to consider the case when $p=\infty$. For a given $j \in\{0,1,2, \ldots, N\}$ consider

$$
I_{j}(\infty):=\max _{t \in \bar{\Omega}}(t(1-t))^{j-\alpha r-r+1}\left|g_{r}^{(j)}(t)\right| .
$$


From (85) and (91) we find

$$
I_{j}(\infty) \leq \sum_{k=0}^{j} \max _{t \in \bar{\Omega}}(t(1-t))^{r(k-\alpha)}\left|U_{k, j}(t)\right| \mid f^{(k)}\left(\gamma_{r}(t) \mid .\right.
$$

Since $U_{k, j} \in C(\bar{\Omega})$ and recalling (92) we have

$$
I_{j}(\infty) \leq c \sum_{k=0}^{j} \max _{t \in \bar{\Omega}}\left(\gamma_{r}(t)\left(1-\gamma_{r}(t)\right)\right)^{k-\alpha}\left|f^{(k)}\left(\gamma_{r}(t)\right)\right| / \Gamma_{r, 0}(t) .
$$

But again we have that $\Gamma_{r, 0} \in C(\bar{\Omega})$ and furthermore does not vanish on $\bar{\Omega}$. On putting $x=\gamma_{r}(t)$ we find from (102) that

$$
I_{j}(\infty) \leq c \sum_{k=0}^{j} \max _{x \in \Omega}(x(1-x))^{k-\alpha}\left|f^{(k)}(x)\right|,
$$

where $c \neq c(f)$. Recalling (2) we have that for $j=0(1) N$

$$
I_{j}(\infty) \leq c\|f\|_{\infty, \alpha, N}
$$

so that $g_{r} \in W_{\infty, \alpha r+r-1}^{(N)}(\Omega)$ with $\left\|g_{r}\right\|_{\infty, \alpha r+r-1, N} \leq c\|f\|_{\infty, \alpha, N}$ which establishes the theorem when $p=\infty$ and completes the proof.

Let us comment on the phrase " $N \in \mathbb{N}$ is sufficiently large" which appears in the statement of Theorem 10. From (84) we see that the functions $g_{r}$ and $f$ need to have the same number of derivatives. Suppose $n_{r} \in \mathbb{N}_{0}$ is such that

$$
n_{r}<r(\alpha+1 / q) \leq n_{r}+1
$$

Recalling the results that have gone before let us then assume that $N \in \mathbb{N}$ is such that

$$
N \geq n_{r}+1,
$$

and this is the value of $N$ that is deemed to be "sufficiently large" in Theorem 10. From Theorem 4(b) we have that if (106) is satisfied then

$$
g_{r}^{(j)}(0)=g_{r}^{(j)}(1)=0 \quad \text { for } \quad j=0(1)\left(n_{r}-1\right) .
$$


On the other hand, if $n<\alpha+1 / q \leq n+1$ then $f$ is such that

$$
f^{(j)}(0)=f^{(j)}(1)=0 \quad \text { for } \quad j=0(1)(n-1) .
$$

Since with our sigmoidal transformations we choose $r>1$ then, in general, we shall have $n_{r}$ bigger than $n$ so that the function $g_{r}$ will in general have more derivatives vanishing at the end points of $\Omega$ than the function $f$ has. This observation will have important consequences when we consider the Euler-Maclaurin quadrature rule for both ordinary and Cauchy principal value integrals in Sections 4 and 5 respectively.

\section{The Euler-Maclaurin formula}

This has been discussed at some length by the author in [4] and we shall both quote results from that paper and use its notation. We let

$$
\text { If }:=\int_{0}^{1} f(x) d x
$$

and if we define

$$
t_{\nu}:=(\nu+1) / 2 \text { for } \quad-1<\nu \leq 1,
$$

then for $m \in \mathbb{N}$ a quadrature rule $Q_{m}^{[\nu]} f$ is defined by

$$
Q_{m}^{[\nu]} f:= \begin{cases}\frac{1}{m} \sum_{j=0}^{m-1} f\left(\left(j+t_{\nu}\right) / m\right), & -1<\nu<1, \\ \frac{1}{m} \sum_{j=0}^{m} f(j / m), & \nu=1,\end{cases}
$$

where $\sum_{j=0}^{m}$ "denotes a sum whose first and last terms are halved. 
For $j \in \mathbb{N}_{0}$, we let $B_{j}$ denote the Bernoulli polynomial of degree $j$; see, for example, Abramowitz and Stegun [1, Chapter 23]. Furthermore, $\bar{B}_{j}$ denotes the periodic extension to $\mathbb{R}$ of $B_{j}$ defined on $[0,1)$. That is,

$$
\bar{B}_{j}(x+k)=B_{j}(x) \quad \text { for all } k \in \mathbb{Z} \quad \text { and } \quad 0 \leq x<1 .
$$

We note that the functions $\bar{B}_{j}$ are continuous on $\mathbb{R}$ except when $j=1$ when it has a finite jump discontinuity at each integer. As a consequence of this and Abramowitz and Stegun [1, $§ 23.1 .5]$ we have that

$$
\bar{B}_{n+1}^{\prime}(x)=(n+1) \bar{B}_{n}(x)
$$

for $n \in \mathbb{N}$ and $x \in \mathbb{R}$.

Our starting point is the following theorem.

Theorem 11 Suppose $f$ is such that for some $s \in \mathbb{N}_{0}, f^{(s)} \in C(\bar{\Omega})$ and $f^{(s+1)} \in L_{1}(\Omega)$. Then, for any $m \in \mathbb{N}$,

$$
\begin{aligned}
E_{m}^{[\nu]} f:=I f-Q_{m}^{[\nu]} f= & -\sum_{j=j_{0}(\nu)}^{s+1} \frac{B_{j}\left(t_{\nu}\right)}{j !} \cdot \frac{f^{(j-1)}(1)-f^{(j-1)}(0)}{m^{j}} \\
& +\frac{1}{m^{s+1}} \int_{0}^{1} f^{(s+1)}(x) \frac{\bar{B}_{s+1}\left(t_{\nu}-m x\right)}{(s+1) !} d x
\end{aligned}
$$

where

$$
j_{0}(\nu):= \begin{cases}1, & |\nu|<1 \\ 2, & \nu=1\end{cases}
$$

Proof: See, for example, Elliott [4, theorem 2.1]. Note that with (114) and (115) we have now corrected the lower limit on the sum to allow for the fact that $Q_{m}^{[1]} f$ incorporates the first term of that sum. 
Note that under the conditions of this theorem we may rewrite (114) as

$$
\begin{aligned}
E_{m}^{[\nu]} f:= & -\sum_{j=j_{0}(\nu)}^{s} \frac{B_{j}\left(t_{\nu}\right)}{j !} \cdot \frac{f^{(j-1)}(1)-f^{(j-1)}(0)}{m^{j}} \\
& +\frac{1}{m^{s+1}} \int_{0}^{1} f^{(s+1)}(x) \frac{\bar{B}_{s+1}\left(t_{\nu}-m x\right)-B_{s+1}\left(t_{\nu}\right)}{(s+1) !} d x .
\end{aligned}
$$

Equations (114) and (116) give the classical forms of the Euler-Maclaurin formula. We shall now consider an upper bound for $\left|E_{m}^{[\nu]} f\right|$ under the assumption that $f$ is in some weighted Sobolev space. But first, a lemma.

Lemma 12 For all $x \in \bar{\Omega}$ and $m, n \in \mathbb{N}$ we have

$$
\left|\bar{B}_{n+1}\left(t_{\nu}-m x\right)-B_{n+1}\left(t_{\nu}\right)\right| \leq c \cdot m x(1-x)
$$

where $c$ is a positive constant depending only on $n$.

Proof: From (113) we have

$$
\begin{aligned}
\left|\bar{B}_{n+1}\left(t_{\nu}-m x\right)-B_{n+1}\left(t_{\nu}\right)\right| & =\left|\bar{B}_{n+1}\left(t_{\nu}\right)-\bar{B}_{n+1}\left(t_{\nu}-m x\right)\right| \\
& =\left|\int_{t_{\nu}-m x}^{t_{\nu}} \bar{B}_{n+1}^{\prime}(\xi) d \xi\right| \\
& =(n+1)\left|\int_{t_{\nu}-m x}^{t_{\nu}} \bar{B}_{n+1}(\xi) d \xi\right| .
\end{aligned}
$$

But, from Abramowitz and Stegun [1, §23.1.16 and §23.2.18], we have that

$$
\max _{x \in \bar{\Omega}}\left|B_{n}(\xi)\right| \leq \begin{cases}1 / 2, & n=1 \\ 2 \zeta(n) n ! /(2 \pi)^{n}, & n \geq 2 .\end{cases}
$$

Consequently from (118) and (119) we have that

$$
\left|\bar{B}_{n+1}\left(t_{\nu}-m x\right)-B_{n+1}\left(t_{\nu}\right)\right| \leq c \cdot m x
$$


where $c=c(n)$. Again we have

$$
\begin{gathered}
\left|\bar{B}_{n+1}\left(t_{\nu}-m x\right)-B_{n+1}\left(t_{\nu}\right)\right|=\left|\bar{B}_{n+1}\left(t_{\nu}-m x\right)-B_{n+1}\left(t_{\nu}-m\right)\right| \\
=\left|\int_{t_{\nu}-m}^{t_{\nu}-m x} \bar{B}_{n+1}^{\prime}(\xi) d \xi\right| \leq c \cdot m(1-x),
\end{gathered}
$$

on using (113) again, where $c=c(n)$. Combining (120) and (121) we obtain (117) and the lemma is proved.

We now come to the starting point of the analysis of an upper bound for $\left|E_{m}^{[\nu]} f\right|$ given that $f$ is in some weighted Sobolev space.

Theorem 13 Suppose $f \in W_{p, \alpha}^{(N)}(\Omega)$ where $1 \leq p \leq \infty, \alpha \in \mathbb{R}, n \in \mathbb{N}_{0}$ and $N \in \mathbb{N}$ are such that

$$
n<\alpha+1 / q \leq n+1 \leq N .
$$

Then, for all $m$ such that $\mathbb{N} \ni m \geq 2$,

$$
E_{m}^{[\nu]} f=\frac{1}{m^{n+1}(n+1) !} \int_{0}^{1} f^{(n+1)}(x)\left(\bar{B}_{n+1}\left(t_{\nu}-m x\right)-B_{n+1}\left(t_{\nu}\right)\right) d x .
$$

Proof: That the integral in (123) exists under the given conditions follows from (117) together with Theorem 7(b). From Theorem 4 we have that $f^{(j)}(0)=f^{(j)}(1)=0$ for $j=0(1)(n-1)$. If in (116), we put $s=n$ we recover (123) and the theorem is proved.

We shall now derive a better rate of convergence of $\left|E_{m}^{[\nu]} f\right|$ to zero than that implied by (117) and (123) which together give that $E_{m}^{[\nu]} f$ tends to zero as $m \rightarrow \infty$ like $\mathcal{O}\left(1 / m^{n}\right)$. 
Theorem 14 Suppose $f \in W_{p, \alpha}^{(N)}(\Omega)$ and $\mathbb{N} \ni m \geq 2$.

(a) If $1 \leq p \leq \infty$ with $n \in \mathbb{N}_{0}$ and $N \in \mathbb{N}$ such that $n<\alpha+1 / q<n+1 \leq N$ then

$$
\left|E_{m}^{[\nu]} f\right| \leq \frac{c\|f\|_{p, \alpha, N}}{m^{\alpha+1 / q}} .
$$

(b) For $1 \leq p \leq \infty$ and $\alpha+1 / q=n+1 \leq N$ then

$$
\left|E_{m}^{[\nu]} f\right| \leq \begin{cases}\frac{c\|f\|_{1, \alpha, N}}{m^{n+1}}, & p=1, \\ \frac{c\|f\|_{p, \alpha, N} \ln ^{1 / q}(m)}{m^{n+1}}, & 1<p \leq \infty .\end{cases}
$$

Here $c$ denotes a positive constant which is independent of both $m$ and $f$.

Proof: From (123) we have

$$
\begin{aligned}
E_{m}^{[\nu]} f=\frac{1}{m^{n+1}(n+1) !} \int_{0}^{1}(x(1-x))^{n+1-\alpha} f^{(n+1)}(x) \times \\
\times \frac{\left(\bar{B}_{n+1}\left(t_{\nu}-m x\right)-B_{n+1}\left(t_{\nu}\right)\right)}{(x(1-x))^{n+1-\alpha}} d x .
\end{aligned}
$$

From Definition 1 (b) (when $p=\infty$ ), the mean value theorem (when $p=1$ ) and Hölder's inequality for $1<p<\infty$ we find

$$
\left|E_{m}^{[\nu]} f\right| \leq \frac{\|f\|_{p, \alpha, N}}{m^{n+1}(n+1) !} \cdot J(p)
$$

say, where the function

$$
J(p):= \begin{cases}\int_{0}^{1} \frac{\left|\bar{B}_{n+1}\left(t_{\nu}-m x\right)-B_{n+1}\left(t_{\nu}\right)\right|}{(x(1-x))^{n+1-\alpha}} d x, & p=\infty, \\ \max _{x \in \bar{\Omega}} \frac{\left|\bar{B}_{n+1}\left(t_{\nu}-m x\right)-B_{n+1}\left(t_{\nu}\right)\right|}{(x(1-x))^{n+1-\alpha}}, & p=1, \\ \left(\int_{0}^{1} \frac{\left|\bar{B}_{n+1}\left(t_{\nu}-m x\right)-B_{n+1}\left(t_{\nu}\right)\right|^{q}}{(x(1-x))^{q(n+1-\alpha)}} d x\right)^{1 / q}, & 1<p<\infty .\end{cases}
$$


First consider the case when $p=1$ so that $1 / q=0$ with $n<\alpha \leq n+1 \leq N$. From this we see that $n+1-\alpha \geq 0$. For $\mathbb{N} \ni m \geq 2$ let us write

$$
\bar{\Omega}=[0,1 / m] \cup[1 / m, 1 / 2] \cup[1 / 2,1-1 / m] \cup[1-1 / m, 1],
$$

and we shall consider the maximum of the quotient on each of these intervals. From (117) we have that on $[0,1 / m]$

$$
\left|\bar{B}_{n+1}\left(t_{\nu}-m x\right)-B_{n+1}\left(t_{\nu}\right)\right| \leq c \cdot m x,
$$

where $c=c(n)$. Consequently

$$
\max _{x \in[0,1 / m]} \frac{\left|\bar{B}_{n+1}\left(t_{\nu}-m x\right)-B_{n+1}\left(t_{\nu}\right)\right|}{(x(1-x))^{n+1-\alpha}} \leq c \cdot m \max _{x \in[0,1 / m]} x^{\alpha-n}=c \cdot m^{n-\alpha+1},
$$

since $\alpha-n>0$. We may argue similarly to give the same upper bound for $x \in[1-1 / m, 1]$. Consider now the interval $[1 / m, 1 / 2]$. From (119)

$$
\max _{x \in[1 / m, 1 / 2]} \frac{\left|\bar{B}_{n+1}\left(t_{\nu}-m x\right)-B_{n+1}\left(t_{\nu}\right)\right|}{(x(1-x))^{n+1-\alpha}} \leq c \cdot \max _{x \in[1 / m, 1 / 2]} \frac{1}{x^{n+1-\alpha}}=c \cdot m^{n-\alpha+1}
$$

where, again, $c=c(n)$. A similar result is to be found over the interval $[1 / 2,1-1 / m]$ so that from (128) and (129) we find

$$
J(1)=\max _{x \in \bar{\Omega}} \frac{\left|\bar{B}_{n+1}\left(t_{\nu}-m x\right)-B_{n+1}\left(t_{\nu}\right)\right|}{(x(1-x))^{n+1-\alpha}} \leq c \cdot m^{n+1-\alpha} .
$$

Consequently from (126) and (130) we see that both (124) and (125) are valid for $p=1$ and $n<\alpha \leq n+1 \leq N$.

Suppose now that $p=\infty$ so $1 / q=1$ and we have $n<\alpha+1 \leq n+1 \leq N$. From (126) let us write

$J(\infty)=\left(\int_{0}^{1 / m}+\int_{1 / m}^{1 / 2}+\int_{1 / 2}^{1-1 / m}+\int_{1-1 / m}^{1}\right) \frac{\left|\bar{B}_{n+1}\left(t_{\nu}-m x\right)-B_{n+1}\left(t_{\nu}\right)\right|}{(x(1-x))^{n+1-\alpha}} d x$. 
From (117) we find, since $0 \leq n-\alpha<1$, that

$$
\int_{0}^{1 / m} \frac{\left|\bar{B}_{n+1}\left(t_{\nu}-m x\right)-B_{n+1}\left(t_{\nu}\right)\right|}{(x(1-x))^{n+1-\alpha}} d x \leq c \cdot m \int_{0}^{1 / m} \frac{d x}{x^{n-\alpha}}=c \cdot m^{n-\alpha},
$$

$c$ being independent of $m$. A similar result holds for the integral over the interval $(1-1 / m, 1)$. For the integral over $(1 / m, 1 / 2)$ we have from (119) that

$$
\begin{aligned}
\int_{1 / m}^{1 / 2} \frac{\left|\bar{B}_{n+1}\left(t_{\nu}-m x\right)-B_{n+1}\left(t_{\nu}\right)\right|}{(x(1-x))^{n+1-\alpha}} d x & \leq c \cdot \int_{1 / m}^{1 / 2} \frac{d x}{x^{n+1-\alpha}} \\
& \leq \begin{cases}c \cdot m^{n-\alpha}, & n-\alpha>0 \\
c \cdot \ln (m), & n=\alpha .\end{cases}
\end{aligned}
$$

Again $c$ is a positive constant independent of $m$. A similar bound is found for the integral over $(1 / 2,1-1 / m)$. To sum up, we have

$$
J(\infty) \leq \begin{cases}c \cdot m^{n-\alpha}, & \text { if } n-\alpha>0, \\ c \cdot \ln (m), & \text { if } n=\alpha .\end{cases}
$$

From (126), (127) and (134) we recover both (124) and (125) in the case when $p=\infty$.

Finally, let us consider the remaining case for which $1<p<\infty$ and $n<\alpha+1 / q \leq n+1 \leq N$. We can argue as we have done in the case when $p=\infty$ by splitting the integral for $J(p)$ in (127) over the four intervals $(0,1 / m),(1 / m, 1 / 2),(1 / 2,1-1 / m),(1-1 / m, 1)$. For the integral over $(0,1 / m)$ we find, from (117) and (127), that

$$
\int_{0}^{1 / m} \frac{\left|\bar{B}_{n+1}\left(t_{\nu}-m x\right)-B_{n+1}\left(t_{\nu}\right)\right|^{q}}{(x(1-x))^{q(n+1-\alpha)}} d x \leq c \cdot m^{q} \int_{0}^{1 / m} \frac{d x}{x^{(n-\alpha) q}}=c \cdot m^{(n+1-\alpha) q-1}
$$


since $(n-\alpha) q<1$. Again, for the integral over $(1 / m, 1 / 2)$ we have on recalling (119) that

$$
\begin{aligned}
& \int_{1 / m}^{1 / 2} \frac{\left|\bar{B}_{n+1}\left(t_{\nu}-m x\right)-B_{n+1}\left(t_{\nu}\right)\right|^{q}}{(x(1-x))^{q(n+1-\alpha)}} d x \\
\leq & c \cdot \int_{1 / m}^{1 / 2} \frac{d x}{x^{(n+1-\alpha) q}} \\
= & \begin{cases}c \cdot m^{(n+1-\alpha) q-1}, & \text { if } \alpha+1 / q<n+1, \\
c \cdot \ln (m), & \text { if } \alpha+1 / q=n+1 .\end{cases}
\end{aligned}
$$

Similar bounds to those given by (135) and (136) are found for the integrals over $(1-1 / m, 1)$ and $(1 / 2,1-1 / m)$ respectively. In all cases $c$ is a positive constant independent of $m$. From (127), (135) and (136), for $1<p<\infty$ we find that

$$
J(p) \leq \begin{cases}c \cdot m^{n+1-\alpha-1 / q}, & \text { if } \alpha+1 / q<n+1, \\ c \cdot \ln ^{1 / q}(m), & \text { if } \alpha+1 / q=n+1 .\end{cases}
$$

From (126) and (137) we see that (124) and (125) follow so that the theorem is proved.

It is appropriate to compare the results of Theorem 14 when $p=1$ with that given in Elliott [5, Theorem 3.3]. There, since $K_{N}^{\alpha}=W_{1, \alpha}^{(N)}(\Omega)$, for $n<\alpha<n+1$ we obtained $\left|E_{m}^{[\nu]} f\right| \leq c\|f\|_{1, \alpha, N} / m^{n}$. For $n<\alpha \leq n+1$ and $p=1$, Theorem 14 gives $\left|E_{m}^{[\nu]} f\right| \leq c\|f\|_{1, \alpha, N} / m^{\alpha}$. The improvement in the rate of convergence of $E_{m}^{[\nu]} f$ to zero is apparent.

Let us now consider the effect of making a sigmoidal transformation to the integral If before using the Euler-Maclaurin formula. That is, we have on writing $x=\gamma_{r}(t)$ in (109) that

$$
I f=\int_{0}^{1} f(x) d x=\int_{0}^{1} f\left(\gamma_{r}(t)\right) \gamma_{r}^{\prime}(t) d t=\int_{0}^{1} g_{r}(t) d t,
$$


on recalling (81). From Theorem 10 we have that if $f \in W_{p, \alpha}^{(N)}(\Omega)$ with $1 \leq p \leq \infty$ then $g_{r} \in W_{p, \alpha r+(r-1) / q}^{(N)}(\Omega)$ and it is to the function $g_{r}$ that we apply the Euler-Maclaurin theorem to give a quadrature rule

$$
I f=I g_{r}=Q_{m}^{[\nu]} g_{r}+E_{m}^{[\nu]} g_{r}=: Q_{m}^{[\nu, r]} f+E_{m}^{[\nu, r]} f,
$$

say. An estimate for $E_{m}^{[\nu]} g_{r}=E_{m}^{[\nu, r]} f$ is given in the following theorem.

Theorem 15 Let $\gamma_{r}$ be a sigmoidal transformation of order $r \geq 1$ and suppose that $f \in W_{p, \alpha}^{(N)}(\Omega)$ with $1 \leq p \leq \infty$. Suppose also that $\mathbb{N} \ni m \geq 2$.

(a) If $1 \leq p \leq \infty$ with $n_{r}, N \in \mathbb{N}$ such that $n_{r}<r(\alpha+1 / q)<n_{r}+1 \leq N$ then

$$
\left|E_{m}^{[\nu, r]} f\right|=\left|E_{m}^{[\nu]} g_{r}\right| \leq \frac{c\|f\|_{p, \alpha, N}}{m^{r(\alpha+1 / q)}} .
$$

(b) For $1 \leq p \leq \infty$ and $r(\alpha+1 / q)=n_{r}+1 \leq N$ we have

$$
\left|E_{m}^{[\nu, r]} f\right|=\left|E_{m}^{[\nu]} g_{r}\right| \leq \begin{cases}\frac{c\|f\|_{1, \alpha, N}}{m^{n_{r}+1}}, & p=1, \\ \frac{c\|f\|_{p, \alpha, N} \ln ^{1 / q}(m)}{m^{n_{r}+1}}, & 1<p \leq \infty .\end{cases}
$$

In both (140) and (141), $c$ is a positive constant which is independent of both $m$ and $f$.

Proof: This follows at once by applying Theorem 14 to the function $g_{r}$ which is in the weighted Sobolev space $W_{p, \alpha r+(r-1) / q}^{(N)}(\Omega)$.

Thus we see that, on comparing (124) with (140), a sigmoidal transformation of order $r$ speeds up the rate of convergence of the quadrature rule to the integral by a factor of $r$. On choosing $r$ to be 4,5 or 6 say, this can lead to a very substantial increase in the rate of convergence. 


\section{Cauchy Principal Value Integrals}

\subsection{Introduction}

Suppose the function $f$ is defined on $\bar{\Omega} \times \Omega$ as follows

$$
f(x ; \lambda):= \begin{cases}\frac{\phi(x)}{x-\lambda}, & x \neq \lambda, \\ 0, & x=\lambda,\end{cases}
$$

where $\phi$ is "well behaved" on $\bar{\Omega}$. (We shall be more explicit about the properties of $\phi$ later.) In order to assign a meaning to $\operatorname{If}(\cdot ; \lambda)$ we shall define the integral as a Cauchy principal value integral; that is we shall write

$$
I f(\cdot ; \lambda)=f_{0}^{1} \frac{\phi(x) d x}{x-\lambda}:=\lim _{\epsilon \rightarrow 0+}\left(\int_{0}^{\lambda-\epsilon}+\int_{\lambda+\epsilon}^{1}\right) \frac{\phi(x) d x}{x-\lambda}
$$

provided that the limit exists. As before, see (111), we shall define $Q_{m}^{[\nu]} f(\cdot ; \lambda)$ by

$$
Q_{m}^{[\nu]} f(\cdot ; \lambda)= \begin{cases}\frac{1}{m} \sum_{j=0}^{m-1} f\left(\left(j+t_{\nu}\right) / m ; \lambda\right), & -1<\nu<1, \\ \frac{1}{m} \sum_{j=0}^{m} f(j / m ; \lambda), & \nu=1 .\end{cases}
$$

We note that since, in(142), we have defined $f(\lambda, \lambda)$ to be zero, $Q_{m}^{[\nu]} f(\cdot ; \lambda)$ is always defined even if, for some integer $l \in\{0,1,2, \ldots, m-1\}$, we have $\left(l+t_{\nu}\right) / m=\lambda$.

In order to obtain a result comparable to Theorem 14, we will introduce an appropriate "subtraction function" $\mathbb{S}_{n}(\cdot ; \lambda)$, see equation (164), with the properties that $\mathbb{S}_{n}(\cdot ; \lambda)$ has the same singularity at $\lambda$ as does the function $f(\cdot ; \lambda)$ and, furthermore, has the same zeros as $\phi$ at the end-points of $\bar{\Omega}$. Then we shall apply Theorem 14 to the function $f(\cdot ; \lambda)-\mathbb{S}_{n}(\cdot ; \lambda)$ and obtain 
both a quadrature rule for the Cauchy principal value integral $\operatorname{If}(\cdot ; \lambda)$ and an estimate of the truncation error $E_{m}^{[\nu]} f(\cdot ; \lambda)$. So much for an outline of the strategy for this section; let us now get down to details. First we require a theorem which will give us an explicit expression for $I-Q_{m}^{[\nu]}$ of certain functions which will play a key role in the development of this theory.

\subsection{The subtraction function}

We shall only prove the next theorem for the case when $-1<\nu<1$, but the modification required when $\nu=1$ is straightforward. In the context of this theorem we define $Q_{m}^{[\nu]} g(\cdot ; \lambda)$ by

$$
Q_{m}^{[\nu]} g(\cdot ; \lambda)= \begin{cases}\frac{1}{m} \sum_{j=0}^{m-1} g\left(\left(j+t_{\nu}\right) / m ; \lambda\right), & m \lambda-t_{\nu} \notin \mathbb{Z}, \\ \frac{1}{m} \sum_{\substack{j=0 \\ j \neq l}}^{m-1} g\left(\left(j+t_{\nu}\right) / m ; \lambda\right), & m \lambda-t_{\nu}=l \in \mathbb{Z} .\end{cases}
$$

Theorem 16 Suppose a function $g(\cdot ; \lambda)$ is defined on the strip $\mathbb{S}$, where $\mathbb{S}:=\{z=x+i y: 0 \leq x \leq 1,-\infty<y<\infty\}$ and satisfies the following conditions:

(a) $g(\cdot ; \lambda)$ is real on $\bar{\Omega}$ and analytic in $\mathbb{S}$ except for a simple pole at the point $\lambda \in \Omega$;

(b) $g(\cdot ; \lambda)$ is 1-periodic;

(c) for $z=x+i a, 0 \leq x \leq 1, m \in \mathbb{N}$ is such that

$$
\lim _{a \rightarrow \infty} e^{-2 \pi m a}|g(x+i a ; \lambda)|=0 \text {. }
$$

Then

$$
I g(\cdot ; \lambda)-Q_{m}^{[\nu]} g(\cdot ; \lambda)=\pi \underset{z=\lambda}{\operatorname{res}}\left\{\cot \left(\pi\left(m z-t_{\nu}\right)\right) g(z ; \lambda)\right\},
$$


where $\underset{z=\lambda}{\operatorname{res}}\{h(z)\}$ denotes the residue of the function $h$ at the point $\lambda$.

Proof: Suppose $-1<\nu<1$. Choose $a>0$ and let $C_{a}$ denote the closed contour described positively (that is, anti-clockwise) comprising the straight line segments joining the points $A(0,-a), B(1,-a), C(1, a), D(0, a)$ and $A$ again. Consider the integral

$$
I_{a}:=\frac{1}{2 \pi i} \int_{C_{a}} g(z ; \lambda) \cot \left(\pi\left(m z-t_{\nu}\right)\right) d z .
$$

If $\lambda \neq\left(l+t_{\nu}\right) / m$ for some integer $l$, the integrand will have simple poles at the points $z_{j}:=\left(j+t_{\nu}\right) / m$, for $j=0(1)(m-1)$, and at the point $\lambda$. For $j=0(1)(m-1)$ we have

$$
\underset{z=z_{j}}{\operatorname{res}}\left\{g(z ; \lambda) \cot \left(\pi\left(m z-t_{\nu}\right)\right)\right\}=\frac{1}{\pi m} g\left(\left(j+t_{\nu}\right) / m ; \lambda\right) .
$$

Applying the residue theorem to $I_{a}$ and recalling (145) we have

$$
I_{a}=\frac{1}{\pi} Q_{m}^{[\nu]} g(\cdot ; \lambda)+\underset{z=\lambda}{\operatorname{res}}\left\{g(z ; \lambda) \cot \left(\pi\left(m z-t_{\nu}\right)\right)\right\} .
$$

Let us now consider the contour integral $I_{a}$ in more detail. We have

$$
\int_{C_{a}}=\int_{A B}+\int_{B C}+\int_{C D}+\int_{D A} .
$$

Since, by condition (b), $g(\cdot ; \lambda)$ is 1-periodic then so is $g(z ; \lambda) \cot \left(\pi\left(m z-t_{\nu}\right)\right)$ so that $\int_{B C}+\int_{D A}$ is zero. Along $A B$ we have $z=x-i a$ with $x$ going from 0 to 1 . Along $C D, z=x+i a$ with $x$ from 1 to 0 . Since the integrand is real when $z$ is real we find

$$
I_{a}=-\frac{1}{\pi} \int_{0}^{1} \Im\left[g(x+i a ; \lambda) \cot \left(\pi\left(m(x+i a)-t_{\nu}\right)\right)\right] d x .
$$


As $\Im(z)=-\Re(i z)$ we find, on writing $x+i a=z$ in (151), that

$$
I_{a}=\frac{1}{\pi} \Re\left\{\int_{i a}^{i a+1} i g(z ; \lambda) \cot \left(\pi\left(m z-t_{\nu}\right)\right) d z\right\} .
$$

From (149) we obtain

$$
\begin{aligned}
Q_{m}^{[\nu]} g(\cdot ; \lambda)= & -\pi \operatorname{res}_{z=\lambda}\left\{g(z ; \lambda) \cot \left(\pi\left(m z-t_{\nu}\right)\right)\right\} \\
& +\Re\left\{\int_{i a}^{i a+1} i g(z ; \lambda) \cot \left(\pi\left(m z-t_{\nu}\right)\right) d z\right\} .
\end{aligned}
$$

Let us now obtain a comparable expression for $\operatorname{Ig}(\cdot ; \lambda)$. Let $C_{a}^{+}$be a contour in the upper half plane $(\Im z>0)$ from $A_{1}(0,0)$ to $B_{1}(1,0)$, but indented at $\lambda$ by a semi-circle $\gamma_{\epsilon}$ say of radius $\epsilon>0$ described negatively, the straight line segments $B_{1} C_{1}$ from $B_{1}(1,0)$ to $C_{1}(1, a), C_{1} D_{1}$ from $C_{1}$ to $D_{1}(0, a)$ and $D_{1} A_{1}$ from $D_{1}$ back to $A_{1}$. Within this closed contour the function $g(\cdot ; \lambda)$ is analytic so that by Cauchy's theorem we have

$$
\int_{C_{a}^{+}} g(z ; \lambda) d z=0
$$

Splitting this integral into its component parts we have, since $g(\cdot ; \lambda)$ is 1 periodic, that $\int_{B_{1} C_{1}}+\int_{D_{1} A_{1}}=0$. From the integrals over $A_{1} B_{1}$ and $C_{1} D_{1}$ we have

$$
\int_{0}^{\lambda-\epsilon} g(x ; \lambda) d x+\int_{\gamma_{\epsilon}} g(z ; \lambda) d z+\int_{\lambda+\epsilon}^{1} g(x ; \lambda) d x+\int_{1+i a}^{i a} g(z ; \lambda) d z=0 .
$$

On letting $\epsilon \rightarrow 0$ and recalling that $\gamma_{\epsilon}$ is a semi-circle described negatively we have

$$
f_{0}^{1} g(x ; \lambda) d x=\pi i \underset{z=\lambda}{\operatorname{res}}\{g(z ; \lambda)\}+\int_{i a}^{1+i a} g(z ; \lambda) d z
$$


On taking complex conjugates and recalling from (a) that $g(\cdot ; \lambda)$ is real on $\bar{\Omega}$ we have simply that

$$
I g(\cdot ; \lambda)=f_{0}^{1} g(x ; \lambda) d x=\Re\left\{\int_{i a}^{i a+1} g(z ; \lambda) d z\right\} .
$$

From (153) and (157) we find

$$
\begin{aligned}
I g(\cdot ; \lambda)-Q_{m}^{[\nu]} g(\cdot ; \lambda)= & \pi \operatorname{res}_{z=\lambda}\left\{g(z ; \lambda) \cot \left(\pi\left(m z-t_{\nu}\right)\right)\right\} \\
& +\Re\left\{\int_{i a}^{i a+1}\left[1-i \cot \left(\pi\left(m z-t_{\nu}\right)\right)\right] g(z ; \lambda) d z\right\} .
\end{aligned}
$$

Consider now the integral over $(i a, i a+1)$ where $a>0$. On writing $z=x+i a$, $0 \leq x \leq 1$, we find

$$
\left|1-i \cot \left(\pi\left(m z-t_{\nu}\right)\right)\right| \leq \frac{2 e^{-2 \pi m a}}{1-e^{-2 \pi m a}} .
$$

Hence

$$
\begin{aligned}
& \Re\left\{\int_{i a}^{i a+1}\left[1-i \cot \left(\pi\left(m z-t_{\nu}\right)\right)\right] g(z ; \lambda) d z\right\} \\
& \leq \frac{2}{1-e^{-2 \pi m a}} \int_{0}^{1} e^{-2 \pi m a}|g(x+i a ; \lambda)| d x .
\end{aligned}
$$

But, from (c), we have that the integral tends to zero in the limit as $a \rightarrow \infty$ so that from (158) we recover (146).

It remains to consider the case when $m \lambda-t_{\nu}=l$ for some integer $l$. In this case we have $\cot \left(\pi\left(m z-t_{\nu}\right)\right)=\cot (\pi m(z-\lambda))$. Consider now, see (147), the integral $I_{a}$. The integrand has simple poles at the points $z_{j}=\left(j+t_{\nu}\right) / m$ for $j=0(1)(m-1)$ with $j \neq l$ and a pole of order two at $\lambda$. Thus we find, recall $(145)$,

$$
I_{a}=(1 / \pi) Q_{m}^{[\nu]} g(\cdot ; \lambda)+\underset{z=\lambda}{\operatorname{res}}\{g(z ; \lambda) \cot (\pi m(z-\lambda))\} .
$$


Arguing as before we find (cf.(153))

$$
\begin{aligned}
Q_{m}^{[\nu]} g(\cdot ; \lambda)= & -\pi \underset{z=\lambda}{\operatorname{res}}\{g(z ; \lambda) \cot (\pi m(z-\lambda))\} \\
& +\Re\left\{\int_{i a}^{i a+1} i g(z ; \lambda) \cot (\pi m(z-\lambda)) d z\right\} .
\end{aligned}
$$

We find from (157) and (162) that

$$
\begin{aligned}
I g(\cdot ; \lambda)-Q_{m}^{[\nu]} g(\cdot ; \lambda)= & \pi \underset{z=\lambda}{\operatorname{res}}\{g(z ; \lambda) \cot (\pi m(z-\lambda))\} \\
& +\Re\left\{\int_{i a}^{i a+1}[1-i \cot (\pi m(z-\lambda))] g(z ; \lambda) d z\right\} .
\end{aligned}
$$

On letting $a \rightarrow \infty$, we can show, as before, that the integral on the right of (163) tends to zero which establishes (146) in this case and the theorem is proved.

Having established this theorem we can now introduce an appropriate subtraction function and apply Theorem 16 to it. We are going to assume that $f(\cdot ; \lambda)$ is defined by (142) and that the function $\phi$ is in the space $W_{p, \alpha}^{(N)}(\Omega)$ where $1 \leq p \leq \infty$ and $n<\alpha+1 / q<n+1 \leq N$ where $n \in \mathbb{N}_{0}$ and $N \in \mathbb{N}$. We know, from Theorem 4 , that $\phi^{(j)}(0)=\phi^{(j)}(1)=0$ for all $j=0(1)(n-1)$. Since $\lambda \in \Omega$, from $(142)$ we have $f^{(j)}(0 ; \lambda)=f^{(j)}(1 ; \lambda)=0$ for $j=0(1)(n-1)$. Again, as $f(\cdot ; \lambda)$ has a pole at $\lambda$, we need to choose the subtraction function $\mathbb{S}_{n}(\cdot ; \lambda)$ so that it both cancels out this singularity and is such that $\mathbb{S}_{n}^{(j)}(0 ; \lambda)=\mathbb{S}_{n}^{(j)}(1 ; \lambda)=0$ for $j=0(1)(n-1)$. Bearing in mind the conditions of Theorem 16 we require also that $\mathbb{S}_{n}(\cdot ; \lambda)$ be 1 -periodic.

Definition 17 For given $\lambda \in \Omega$ and $n \in \mathbb{N}_{0}$, the subtraction function $\mathbb{S}_{n}(\cdot ; \lambda)$ is defined on $\bar{\Omega} \times \Omega$ by

$$
\mathbb{S}_{n}(x ; \lambda):=\frac{\sin ^{n}(\pi x)}{\sin ^{n}(\pi \lambda)}\left\{\begin{array}{lll}
\pi \cot (\pi(x-\lambda)), & x \neq \lambda, & n \text { even } \\
\pi \csc (\pi(x-\lambda)), & x \neq \lambda, & n \text { odd } \\
0, & x=\lambda .
\end{array}\right.
$$


Note in passing that when $n=0, \mathbb{S}_{0}(\cdot ; \lambda)$ corresponds to the subtraction function $\psi$ introduced by Lyness [8].

Theorem 18 For a given $n \in \mathbb{N}_{0}$ and for all $m \in \mathbb{N}$ such that $m \geq[n / 2]+1$ we have

$$
I \mathbb{S}_{n}(\cdot ; \lambda)-Q_{m}^{[\nu]} \mathbb{S}_{n}(\cdot ; \lambda)= \begin{cases}\pi \cot \left(\pi\left(m \lambda-t_{\nu}\right)\right), & m \lambda-t_{\nu} \notin \mathbb{Z} \\ (\pi n / m) \cot (\pi \lambda), & m \lambda-t_{\nu} \in \mathbb{Z}\end{cases}
$$

Proof: We may, from (164), continue the definition of $\mathbb{S}_{n}(x ; \lambda)$ into the strip $\mathbb{S}$ by replacing $x$ by $z$ but ignoring the condition in $(164)$ that $\mathbb{S}_{n}(\lambda ; \lambda)=$ 0 . Instead we observe that for $n$ both even and odd the function $\mathbb{S}_{n}(z ; \lambda)$ has a simple pole at $z=\lambda$ with residue 1 . With this $\mathbb{S}_{n}(z ; \lambda)$, it is readily verified that it satisfies conditions (a) and (b) of Theorem 16. Let us now consider condition (c).

When $n$ is even we need to determine

$$
\lim _{a \rightarrow \infty} e^{-2 \pi m a}\left|\pi \frac{\sin ^{n}(\pi(x+i a))}{\sin ^{n}(\pi \lambda)} \cot (\pi(x+i a-\lambda))\right|,
$$

where $0 \leq x \leq 1$. From Abramowitz and Stegun [1, §4.3.83]

$$
\left|\sin ^{n}(\pi(x+i a))\right| \leq \cosh ^{n}(\pi a) .
$$

Since

$$
\cot (\pi(x+i a-\lambda))=-i \frac{1+e^{-2 \pi a} e^{2 i \pi(x-\lambda)}}{1-e^{-2 \pi a} e^{2 i \pi(x-\lambda)}},
$$

it follows that

$$
\lim _{a \rightarrow \infty}|\cot (\pi(x+i a-\lambda))|=1 .
$$

From (166), (167) and (169) we see that condition (c) of Theorem 16 will be satisfied if $m$ is such that $m \geq n / 2+1$. 
Suppose now that $n$ is odd. From $[1, \S 4.3 .85]$ we have

$$
|\csc (\pi(x+i a-\lambda))| \leq 1 / \sinh (\pi a) .
$$

Then, from (164), (167) and (170) we have that

$$
\lim _{a \rightarrow \infty} e^{-2 \pi m a}\left|\mathbb{S}_{n}(x+i a ; \lambda)\right|=0,
$$

provided that $-2 m+n-1<0$. Since $n$ is odd this will be true when $m \geq[n / 2]+1$.

Thus, under the given conditions, we can apply Theorem 16 to the function $\mathbb{S}_{n}(\cdot ; \lambda)$ to give

$$
I \mathbb{S}_{n}(\cdot ; \lambda)-Q_{m}^{[\nu]} \mathbb{S}_{n}(\cdot ; \lambda)=\pi \underset{z=\lambda}{\operatorname{res}}\left\{\cot \left(\pi\left(m z-t_{\nu}\right)\right) \mathbb{S}_{n}(z ; \lambda)\right\}
$$

We need to consider the residue at $\lambda$ in the two cases when $m \lambda-t_{\nu} \notin \mathbb{Z}$ and $m \lambda-t_{\nu}=l$ where $l \in\{0,1, \ldots, m-1\}$.

Let us recall from $[1, \S 4.3 .68$ and $\S 4.3 .70]$ that

$$
\pi\left\{\begin{array}{l}
\cot \\
\csc
\end{array}\right\}(\pi(z-\lambda))=\frac{1}{z-\lambda}+\mathcal{O}(z-\lambda) .
$$

When $m \lambda-t_{\nu} \notin \mathbb{Z}$ the function $\cot \left(\pi\left(m z-t_{\nu}\right)\right) \mathbb{S}_{n}(z ; \lambda)$ has a simple pole at $z=\lambda$ and we have, for $n$ both odd and even, that

$$
\pi \underset{z=\lambda}{\operatorname{res}}\left\{\cot \left(\pi\left(m z-t_{\nu}\right)\right) \mathbb{S}_{n}(z ; \lambda)\right\}=\pi \cot \left(\pi\left(m \lambda-t_{\nu}\right)\right) .
$$

This establishes the first of (165).

Suppose now that $m \lambda-t_{\nu}=l$, an integer such that $l \in\{0,1, \ldots, m-1\}$. For $z \neq \lambda$ it follows that $\cot \left(\pi\left(m z-t_{\nu}\right)\right)=\cot (\pi m(z-\lambda))$. In this case the function $\cot (\pi m(z-\lambda)) \mathbb{S}_{n}(z ; \lambda)$ has a pole of order two at $z=\lambda$ for $n$ both 
even and odd. Since, near $z=0$, both $\cot z$ and $\csc z$ behave like $1 / z+\mathcal{O}(z)$ we find near $z=\lambda$, for $n$ either even or odd that

$$
\mathbb{S}_{n}(z ; \lambda)=\frac{1}{z-\lambda}+n \pi \cot (\pi \lambda)+\mathcal{O}(z-\lambda) .
$$

It then follows that near $z=\lambda$

$$
\cot (\pi m(z-\lambda)) \mathbb{S}_{n}(z ; \lambda)=\frac{1}{\pi m(z-\lambda)^{2}}+\frac{n}{m} \cot (\pi \lambda) \frac{1}{z-\lambda}+\mathcal{O}(1),
$$

and we find

$$
\pi \underset{z=\lambda}{\operatorname{res}}\left\{\cot (\pi m(z-\lambda)) \mathbb{S}_{n}(z ; \lambda)\right\}=(\pi n / m) \cot (\pi \lambda) .
$$

This is the second of (165) and the theorem is proved.

We comment in passing that (165) is a very neat result giving the difference, between the Cauchy principal value integral $I \mathbb{S}_{n}(\cdot ; \lambda)$ and the quadrature sum $Q_{m}^{[\nu]} \mathbb{S}_{n}(\cdot ; \lambda)$, as a single term. This generalises beautifully a result given by Lyness [8]. We also note that the right-hand side of (165) does not depend on whether $n$ is even or odd although of course, the definition of $\mathbb{S}_{n}(\cdot ; \lambda)$ does.

\subsection{The function $f_{n}$}

In order to use the results of Section 4 to obtain the appropriate quadrature rule for Cauchy principal value integrals we shall now introduce a function $f_{n}$ say, which satisfies the conditions of Theorem 14. We define, on $\bar{\Omega} \times \Omega$,

$$
f_{n}(x ; \lambda):= \begin{cases}\frac{\phi(x)}{x-\lambda}-\phi(\lambda) \mathbb{S}_{n}(x ; \lambda), & x \neq \lambda, \\ \phi^{\prime}(\lambda)-n \pi \phi(\lambda) \cot (\pi \lambda), & x=\lambda .\end{cases}
$$

Note that $f_{n}(\lambda ; \lambda)$ is defined as $\lim _{x \rightarrow \lambda} f_{n}(x ; \lambda)$ and the definition in (178) follows from (164), (175) and the application of l'Hôpital's rule. 
From (164), for $x \neq \lambda$ we may write $\mathbb{S}_{n}$ as

$$
\mathbb{S}_{n}(x ; \lambda)=\frac{\sin ^{n}(\pi x)}{\sin ^{n}(\pi \lambda)}\left[\frac{1}{x-\lambda}+h_{n}(x ; \lambda)\right],
$$

say where, from Abramowitz and Stegun [1, $§ 4.3 .91$ and $\S 4.3 .93]$, the function

$$
h_{n}(x ; \lambda):=\sum_{k=1}^{\infty} \frac{2 a_{k, n}(x-\lambda)}{(x-\lambda)^{2}-k^{2}},
$$

for $|x-\lambda|<1$, the coefficients $a_{k, n}$ being defined by

$$
a_{k, n}:= \begin{cases}1, & n \text { even } \\ (-1)^{k}, & n \text { odd }\end{cases}
$$

From (178) to (180) we shall write $f_{n}$ as

$$
f_{n}(x ; \lambda)=f_{n, 1}(x ; \lambda)-f_{n, 2}(x ; \lambda),
$$

where $f_{n, 1}$ and $f_{n, 2}$ are defined on $\bar{\Omega} \times \Omega$ by

$$
f_{n, 1}(x ; \lambda):= \begin{cases}\sin ^{n}(\pi x)\left(\frac{\phi(x) \csc ^{n}(\pi x)-\phi(\lambda) \csc ^{n}(\pi \lambda)}{x-\lambda}\right), & x \neq \lambda, \\ \phi^{\prime}(\lambda)-n \pi \phi(\lambda) \cot (\pi \lambda), & x=\lambda ;\end{cases}
$$

and

$$
f_{n, 2}(x ; \lambda):=\phi(\lambda) \csc ^{n}(\pi \lambda) \sin ^{n}(\pi x) h_{n}(x ; \lambda) .
$$

Given that $\phi$ is in some appropriate weighted Sobolev space we now need to find the corresponding space for $f_{n}$ before applying Theorem 14. First, the function $f_{n, 1}(\cdot ; \lambda)$.

Theorem 19 For $n \in \mathbb{N}_{0}, \mathbb{N} \ni N \geq 2$ and $1 \leq p \leq \infty$, suppose that $\phi \in W_{p, \alpha}^{(N)}(\Omega)$ where

$$
n<\alpha+1 / q<n+1 \leq N .
$$


(a) If $p=\infty$, then $f_{n, 1}(\cdot ; \lambda) \in W_{\infty, \alpha}^{(N-1)}(\Omega)$ with

$$
\left\|f_{n, 1}(\cdot ; \lambda)\right\|_{\infty, \alpha, N-1} \leq \frac{c\|\phi\|_{\infty, \alpha, N}}{\lambda(1-\lambda)}
$$

where $c$ is independent of $\lambda$ and $\phi$.

(b) If $1 \leq p<\infty$, then $f_{n, 1}(\cdot ; \lambda) \in W_{p, \alpha-\epsilon}^{(N-2)}(\Omega)$ for any positive $\epsilon$ (no matter how small) with

$$
\left\|f_{n, 1}(\cdot ; \lambda)\right\|_{p, \alpha-\epsilon, N-2} \leq \frac{c\|\phi\|_{p, \alpha, N}}{\lambda(1-\lambda)}
$$

where $c$ is independent of $\lambda$ and $\phi$.

Proof: For $x \in \Omega$ and $n \in \mathbb{N}_{0}$ let us write

$$
\psi_{n}(x):=\phi(x) \csc ^{n}(\pi x) .
$$

Now the function $\csc ^{n}(\pi x)$ is defined on $\Omega$ and is infinitely differentiable there. Since, for $x \in \bar{\Omega}$,

$$
\pi x(1-x) \leq \sin (\pi x) \leq 4 x(1-x),
$$

we conclude, by considering the behaviour of $\csc ^{n}(\pi x)$ near its end-points, that it is in the space $W_{\infty,-n}^{(N)}(\Omega)$ for any $N \in \mathbb{N}_{0}$ and its norm will depend only on $n$ and $N$. By Theorem 8 we will have that $\psi_{n} \in W_{p, \alpha-n}^{(N)}(\Omega)$ for $1 \leq p \leq \infty$ and furthermore

$$
\left\|\psi_{n}\right\|_{p, \alpha-n, N} \leq c\|\phi\|_{p, \alpha, N} .
$$

(a) Suppose $p=\infty$. If we write

$$
\Psi_{n}(x ; \lambda):=\frac{\psi_{n}(x ; \lambda)-\psi_{n}(\lambda ; \lambda)}{x-\lambda},
$$


then, by Theorem $26, \Psi_{n}(\cdot ; \lambda) \in W_{\infty, \alpha-n}^{(N-1)}(\Omega)$ and

$$
\left\|\Psi_{n}(\cdot ; \lambda)\right\|_{\infty, \alpha-n, N-1} \leq \frac{c\left\|\psi_{n}\right\|_{\infty, \alpha-n, N-1}}{\lambda(1-\lambda)}
$$

where $c$ is independent of $\lambda$ and $\psi_{n}$. Since $\sin ^{n}(\pi x) \in W_{\infty, n}^{(N-1)}(\Omega)$ it follows from (183) and Theorem 8 again that $f_{n, 1}(\cdot ; \lambda) \in W_{\infty, \alpha}^{(N-1)}(\Omega)$ and furthermore, from (190), that (186) is satisfied.

(b) Suppose $1 \leq p<\infty$. From Theorem 27 applied to the function $\psi_{n}$ we have, for any $\epsilon>0$, that $\Psi_{n}(\cdot ; \lambda) \in W_{p, \alpha-n-\epsilon}^{(N-2)}(\Omega)$ with

$$
\left\|\Psi_{n}(\cdot ; \lambda)\right\|_{p, \alpha-n-\epsilon, N-2} \leq \frac{c\left\|\psi_{n}\right\|_{p, \alpha-n, N}}{\lambda(1-\lambda)} \leq \frac{c\|\phi\|_{p, \alpha, N}}{\lambda(1-\lambda)}
$$

on using (190) where $c$ is independent of both $\lambda$ and $\phi$. Again, since $\sin ^{n}(\pi x) \in W_{\infty, n}^{(N-2)}(\Omega)$ we have from Theorem 8 applied to (183) that $f_{n, 1}(\cdot ; \lambda) \in W_{p, \alpha-\epsilon}^{(N-2)}(\Omega)$ with its norm satisfying (187).

Consider now the function $f_{n, 2}(\cdot ; \lambda)$, see $(184)$. Before stating the result, we first need the following property of the function $h_{n}(\cdot ; \lambda)$, see (180) and (181).

Theorem 20 For all $n \in \mathbb{N}_{0}, \lambda \in \Omega$ and $N \in \mathbb{N}$ we have that

$$
h_{n}(\cdot ; \lambda) \in W_{\infty, 0}^{(N-1)}(\Omega),
$$

with

$$
\left\|h_{n}(\cdot ; \lambda)\right\|_{\infty, 0, N-1} \leq \frac{c}{\lambda(1-\lambda)},
$$

where $c$ is a positive constant independent of $\lambda$. 
Proof: For $j=\{0,1, \ldots, N-1\}$ and given $\lambda \in \Omega$ we need to consider

$$
H_{j}(\lambda):=\max _{0 \leq x \leq 1}(x(1-x))^{j}\left|h_{n}^{(j)}(x ; \lambda)\right| .
$$

We first observe from (180) that

$$
h_{n}(1-x ; \lambda)=-h_{n}(x ; 1-\lambda),
$$

so that for $j=0(1)(N-1)$

$$
h_{n}^{(j)}(1-x ; \lambda)=(-1)^{j+1} h_{n}^{(j)}(x ; 1-\lambda) .
$$

As a consequence we see from (195) that we may write

$$
H_{j}(\lambda)=\max \left\{H_{j, 1}(\lambda), H_{j, 1}(1-\lambda)\right\}
$$

say, where we define

$$
H_{j, 1}(\lambda):=\max _{0 \leq x \leq \lambda}(x(1-x))^{j}\left|h_{n}^{(j)}(x ; \lambda)\right| .
$$

Suppose that $j=0$; from (180)

$$
\left|h_{n}(x ; \lambda)\right| \leq 2 \sum_{k=1}^{\infty} \frac{1}{k^{2}-(x-\lambda)^{2}} .
$$

Since for $0 \leq x \leq \lambda$ we have $k^{2}-\lambda^{2} \leq k^{2}-(x-\lambda)^{2}$ it follows that

$$
H_{0,1}(\lambda) \leq 2\left\{\frac{1}{1-\lambda^{2}}+\sum_{k=2}^{\infty} \frac{1}{k^{2}-\lambda^{2}}\right\}
$$

Since, for $k \geq 2,(k-1)^{2} \leq k^{2}-\lambda^{2}$ we have, from the definition of the Riemann zeta function (see Abramowitz and Stegun [1, §23.2.1]), that

$$
H_{0,1}(\lambda) \leq 2\left\{\frac{1}{1-\lambda^{2}}+\zeta(2)\right\} \leq \frac{c}{1-\lambda},
$$


where $c$ is independent of $\lambda$. From (198) we have that

$$
H_{0}(\lambda) \leq c \max \left\{\frac{1}{1-\lambda}, \frac{1}{\lambda}\right\} \leq \frac{c}{\lambda(1-\lambda)} .
$$

We may proceed similarly for $H_{j}(\lambda)$ when $j=1(1)(N-1)$. From (180), on splitting each term of the sum into partial fractions and differentiating $j$ times we find that

$$
H_{j, 1}(\lambda) \leq j ! \max _{0 \leq x \leq \lambda}\left\{\sum_{k=1}^{\infty} \frac{(x(1-x))^{j}}{(k-\lambda+x)^{j+1}}+\sum_{k=1}^{\infty} \frac{(x(1-x))^{j}}{(k+\lambda-x)^{j+1}}\right\} .
$$

For $0 \leq x \leq \lambda$ we have that

$$
k-1 \leq k-\lambda+x \quad \text { and } \quad k \leq k+\lambda-x,
$$

so that

$$
H_{j, 1}(\lambda) \leq j !\left\{\max _{0 \leq x \leq \lambda} \frac{(x(1-x))^{j}}{(1-\lambda+x)^{j+1}}+2 \zeta(j+1)\right\},
$$

on recalling the definition of the Riemann zeta function. It readily follows that

$$
\max _{0 \leq x \leq \lambda} \frac{(x(1-x))^{j}}{(1-\lambda+x)^{j+1}} \leq \max _{0 \leq x \leq \lambda} \frac{1}{1-\lambda+x} \times \max _{0 \leq x \leq \lambda}\left(\frac{x(1-x))}{1-\lambda+x}\right)^{j} \leq \frac{1}{1-\lambda} .
$$

From (205) and (206) we have

$$
H_{j, 1}(\lambda) \leq \frac{c}{1-\lambda}
$$

for some $c$ which is independent of $\lambda$. From (198) we have that

$$
H_{j}(\lambda) \leq c \max \left\{\frac{1}{\lambda}, \frac{1}{1-\lambda}\right\}
$$

so that (194) follows at once and the theorem is proved.

We are now in a position to determine the weighted Sobolev space in which the function $f_{n, 2}(\cdot ; \lambda)$ may be found. 
Theorem 21 Suppose $\phi \in W_{p, \alpha}^{(N)}(\Omega)$ for $1 \leq p \leq \infty$ with $n \in \mathbb{N}_{0}$ and $N \in \mathbb{N}$ such that

$$
n<\alpha+1 / q<n+1 \leq N .
$$

Then $f_{n, 2}(\cdot ; \lambda) \in W_{\infty, n}^{(N-1)}(\Omega)$ with

$$
\left\|f_{n, 2}(\cdot ; \lambda)\right\|_{\infty, n, N-1} \leq \frac{c\|\phi\|_{p, \alpha, N}}{(\lambda(1-\lambda))^{n+2-(\alpha+1 / q)}},
$$

where $c$ is independent of $\lambda$ and $\phi$.

Proof: Consider the function

$$
s_{n}(x):=\sin ^{n}(\pi x) .
$$

This is infinitely differentiable on $\Omega$ and, from considering its behaviour near the end-points of $\Omega$, we have that $s_{n} \in W_{\infty, n}^{(N-1)}(\Omega)$ with $\left\|s_{n}\right\|_{\infty, n, N-1} \leq c$, with $c$ depending only on $n$ and $N$. By Theorems 8 and 20 it follows that $s_{n} h_{n}(\cdot ; \lambda) \in W_{\infty, n}^{(N-1)}(\Omega)$ with

$$
\left\|s_{n} h_{n}(\cdot ; \lambda)\right\|_{\infty, n, N-1} \leq \frac{c}{\lambda(1-\lambda)}
$$

where $c$ is independent of $\lambda$. From (184) it follows that $f_{n, 2}(\cdot ; \lambda) \in W_{\infty, n}^{(N-1)}(\Omega)$.

Since $\phi \in W_{p, \alpha}^{(N)}(\Omega)$ it follows from (21) that

$$
|\phi(\lambda)| \leq c\|\phi\|_{p, \alpha, N}(\lambda(1-\lambda))^{\alpha-1 / p},
$$

where $c$ is independent of $\lambda$. Again from (189) we have that for some $c$ independent of $\lambda$

$$
\csc ^{n}(\pi \lambda) \leq c\left(\lambda(1-\lambda)^{-n}\right.
$$

From (3), (184), (213) and (214) it follows that (210) follows immediately and the theorem is proved. 
Comment: as a consequence of (209), inequality (210) may be replaced by the weaker result that

$$
\left\|f_{n, 2}(\cdot ; \lambda)\right\|_{\infty, n, N-1} \leq \frac{c\|\phi\|_{p, \alpha, N}}{(\lambda(1-\lambda))^{2}},
$$

and this will be useful later when we consider sigmoidal transformations.

By combining the results of Theorem 19 and 21 we now identify a suitable weighted Sobolev space for the function $f_{n}(\cdot ; \lambda)$, see $(182)$, and also its norm in that space.

Theorem 22 Suppose $\phi \in W_{p, \alpha}^{(N)}(\Omega)$ for $1 \leq p \leq \infty$, with $n \in \mathbb{N}_{0}$ and $\mathbb{N} \ni N \geq 2$ such that

$$
n<\alpha+1 / q<n+1 \leq N .
$$

(a) If $p=\infty$, then $f_{n}(\cdot ; \lambda) \in W_{\infty, \alpha}^{(N-1)}(\Omega)$ with

$$
\left\|f_{n}(\cdot ; \lambda)\right\|_{\infty, \alpha, N-1} \leq \frac{c\|\phi\|_{\infty, \alpha, N}}{(\lambda(1-\lambda))^{n+1-\alpha}},
$$

where $c$ is independent of $\phi$ and $\lambda$.

(b) If $1 \leq p<\infty$, then $f_{n}(\cdot ; \lambda) \in W_{p, \alpha-\epsilon}^{(N-2)}(\Omega)$ for any $\epsilon>0$ with

$$
\left\|f_{n}(\cdot ; \lambda)\right\|_{p, \alpha-\epsilon, N-2} \leq \frac{c\|\phi\|_{p, \alpha, N}}{(\lambda(1-\lambda))^{n+2-(\alpha+1 / q)}},
$$

$c$ being independent of $\phi$ and $\lambda$. 


\section{Proof:}

(a) Suppose $p=\infty$. Theorem 19(a) implies that $f_{n, 1}(\cdot ; \lambda) \in W_{\infty, \alpha}^{(N-1)}(\Omega)$ and from Theorem 21 we have that $f_{n, 2}(\cdot ; \lambda) \in W_{\infty, n}^{(N-1)}(\Omega)$. Since from (216) when $p=\infty$ we have $1 / q=1$ it follows that $\alpha<n$ so that by Theorem 2(b) we have $f_{n, 2}(\cdot ; \lambda) \in W_{\infty, \alpha}^{(N-1)}(\Omega)$ and furthermore

$$
\left\|f_{n, 2}(\cdot ; \lambda)\right\|_{\infty, \alpha, N-1} \leq c\left\|f_{n, 2}(\cdot ; \lambda)\right\|_{\infty, n, N-1} .
$$

On recalling (182) we have that $f_{n}(\cdot ; \lambda) \in W_{\infty, \alpha}^{(N-1)}(\Omega)$ and from (186), (210) and (219), (217) follows since $n+1-\alpha>1$.

(b) Suppose now that $1 \leq p<\infty$. For any positive $\epsilon$ we have from Theorem 19(b) that $f_{n, 1}(\cdot ; \lambda) \in W_{p, \alpha-\epsilon}^{(N-2)}(\Omega)$ with norm given by (187). Again, from Theorem 21 we have $f_{n, 2}(\cdot ; \lambda) \in W_{\infty, n}^{(N-1)}(\Omega)$ with norm given by (210). However, Theorem 6 implies that $f_{n, 2}(\cdot ; \lambda) \in W_{p, \alpha-\epsilon}^{(N-1)}(\Omega)$ with

$$
\left\|f_{n, 2}(\cdot ; \lambda)\right\|_{p, \alpha-\epsilon, N-1} \leq c\left\|f_{n, 2}(\cdot ; \lambda)\right\|_{\infty, n, N-1},
$$

for some $c$ independent of $f_{n, 2}$. From (182) we shall certainly have $f_{n}(\cdot ; \lambda) \in W_{p, \alpha-\epsilon}^{(N-2)}(\Omega)$ and from (182), (187), (210), (216) and (220) we see that (218) follows and the theorem is proved.

We are now in a position to apply Theorem 14(a) to the function $f_{n}(\cdot ; \lambda)$ which will give the required quadrature rule with error term for the Cauchy principal value integral $\operatorname{If}(\cdot ; \lambda)$. This we shall do in the next section.

\subsection{The denouement}

Let us recall that the function $f(\cdot ; \lambda)$ is defined in (142), the Cauchy principal value integral $\operatorname{If}(\cdot ; \lambda)$ in (143) and the sum $Q_{m}^{[\nu]} f(\cdot ; \lambda)$ in (144). It 
is appropriate at this stage, following Lyness [8, eq(1.12)], to define the function

$$
S_{m}^{[\nu]} f(\cdot ; \lambda):= \begin{cases}\pi \phi(\lambda) \cot \left(\pi\left(t_{\nu}-m \lambda\right)\right), & m \lambda-t_{\nu} \notin \mathbb{Z}, \\ -\phi^{\prime}(\lambda) / m, & m \lambda-t_{\nu} \in \mathbb{Z} .\end{cases}
$$

We now have the following theorem.

Theorem 23 Suppose $\phi \in W_{p, \alpha}^{(N)}(\Omega)$ where $1 \leq p \leq \infty, \mathbb{N} \ni N \geq 2$ and $\alpha$ is such that

$$
n<\alpha+1 / q<n+1 \leq N,
$$

for some $n \in \mathbb{N}_{0}$. For $m \in \mathbb{N}$ such that $m \geq[n / 2]+1$ and $\lambda \in \Omega$ we have

$$
I f(\cdot ; \lambda)=f_{0}^{1} \frac{\phi(x) d x}{x-\lambda}=Q_{m}^{[\nu]} f(\cdot ; \lambda)-S_{m}^{[\nu]} f(\cdot ; \lambda)+E_{m}^{[\nu]} f(\cdot ; \lambda)
$$

where the truncation error $E_{m}^{[\nu]} f(\cdot ; \lambda)$ is such that

$$
\left|E_{m}^{[\nu]} f(\cdot ; \lambda)\right| \leq \frac{c\|\phi\|_{p, \alpha, N}}{(\lambda(1-\lambda))^{n+2-(\alpha+1 / q)} m^{\alpha+1 / q-\epsilon(p)}},
$$

$c$ being independent of $\lambda, \phi$ and $m$ and $\epsilon(p)$ being such that $\epsilon(\infty)=0$ and $\epsilon(p)>0$ for $1 \leq p<\infty$.

Proof: Suppose first that $m \lambda-t_{\nu} \notin \mathbb{Z}$. Applying Theorem 14 to the function $f_{n}(\cdot ; \lambda)$ we have from

$$
I f_{n}(\cdot ; \lambda)=Q_{m}^{[\nu]} f_{n}(\cdot ; \lambda)+E_{m}^{[\nu]} f_{n}(\cdot ; \lambda)
$$

that

$$
I f(\cdot ; \lambda)=Q_{m}^{[\nu]} f(\cdot ; \lambda)+\phi(\lambda)\left(I \mathbb{S}_{n}(\cdot ; \lambda)-Q_{m}^{[\nu]} \mathbb{S}_{n}(\cdot ; \lambda)\right)+E_{m}^{[\nu]} f_{n}(\cdot ; \lambda) .
$$

From Theorem 18 we immediately recover $(223)$ with $S_{m}^{[\nu]} f(\cdot ; \lambda)$ being defined by (221). 
Suppose now that $m \lambda-t_{\nu}=l$ for some $l \in\{0,1, \ldots, m-1\}$ and $|\nu|<1$. We now have

$$
I f_{n}(\cdot ; \lambda)=\frac{1}{m} \sum_{\substack{j=0 \\ j \neq l}}^{m-1} f_{n}\left(\frac{j+t_{\nu}}{m} ; \lambda\right)+\frac{1}{m} f_{n}(\lambda ; \lambda)+E_{m}^{[\nu]} f_{n}(\cdot ; \lambda) .
$$

From (178) we obtain

$$
\begin{aligned}
I f(\cdot ; \lambda)= & \frac{1}{m} \sum_{\substack{j=0 \\
j \neq l}}^{m-1} f_{n}\left(\frac{j+t_{\nu}}{m} ; \lambda\right)-\frac{\phi(\lambda)}{m} \sum_{\substack{j=0 \\
j \neq l}}^{m-1} \mathbb{S}_{n}\left(\frac{j+t_{\nu}}{m} ; \lambda\right)+\phi(\lambda) I \mathbb{S}_{n}(\cdot ; \lambda) \\
& +\frac{1}{m}\left\{\phi^{\prime}(\lambda)-n \pi \phi(\lambda) \cot (\pi \lambda)\right\}+E_{m}^{[\nu]} f_{n}(\cdot ; \lambda) .
\end{aligned}
$$

Recall, from (142), that $f\left(\left(l+t_{\nu}\right) / m ; \lambda\right)=f(\lambda ; \lambda)=0$ and, from (164), that $\mathbb{S}_{n}\left(\left(l+t_{\nu}\right) / m ; \lambda\right)=\mathbb{S}_{n}(\lambda ; \lambda)=0$. Restoring these zero terms to the sums gives

$$
\begin{aligned}
I f(\cdot ; \lambda)= & Q_{m}^{[\nu]} f(\cdot ; \lambda)+\phi(\lambda)\left\{I \mathbb{S}_{n}(\cdot ; \lambda)-Q_{m}^{[\nu]} \mathbb{S}_{n}(\cdot ; \lambda)\right\} \\
& +\frac{1}{m}\left\{\phi^{\prime}(\lambda)-n \pi \phi(\lambda) \cot (\pi \lambda)\right\}+E_{m}^{[\nu]} f_{n}(\cdot ; \lambda) .
\end{aligned}
$$

But, from (165) and (221), since $m \lambda-t_{\nu} \in \mathbb{Z}$ we recover (223) again.

It remains to put an upper bound on $\left|E_{m}^{[\nu]} f_{n}(\cdot ; \lambda)\right|$. If, when $1 \leq p<\infty$ we apply Theorem 14(a) to the function $f_{n}(\cdot ; \lambda)$ which, from Theorem 22(b), we know to be in $W_{p, \alpha-\epsilon}^{(N-2)}(\Omega)$ we have from (124) and (218) that

$$
\left|E_{m}^{[\nu]} f_{n}(\cdot ; \lambda)\right| \leq \frac{c\|\phi\|_{p, \alpha, N}}{(\lambda(1-\lambda))^{n+2-(\alpha+1 / q)} m^{\alpha+1 / q-\epsilon}} .
$$

Alternatively when $p=\infty$ we know that $f_{n}(\cdot ; \lambda) \in W_{\infty, \alpha}^{(N-1)}(\Omega)$, from Theorem 22(a), with norm given by (217). This and (124) together give

$$
\left|E_{m}^{[\nu]} f_{n}(\cdot ; \lambda)\right| \leq \frac{c\|\phi\|_{\infty, \alpha, N}}{(\lambda(1-\lambda))^{n+1-\alpha} m^{\alpha+1}} .
$$


In both (230) and (231) the constant $c$ is independent of $\lambda, \phi$ and $m$. These two results may be combined as in (224), and the theorem is proved.

Note: in spite of the complexity of the subtraction function for arbitrary $n \in \mathbb{N}_{0}$ we have obtained the same quadrature rule as did Lyness [8] for the particular case where $n=0$. We also comment from (224) that for $1 \leq p<\infty$ the rate of convergence is $\mathcal{O}\left(1 / m^{\alpha+1 / q-\epsilon}\right)$, where we may choose $\epsilon$ to be arbitrarily small. We have been unable to derive a similar result with $\epsilon=0$. Finally we comment on the dependence of the error on $\lambda$. When $\lambda$ is close to either end-point the term $1 /(\lambda(1-\lambda))^{n+2-(\alpha+1 / q)}$ will become large but we note that the exponent of $\lambda(1-\lambda))$ is always less than 2 , independent of the value of $n$. This will be of some importance when, in the next section, we consider sigmoidal transformations.

\subsection{Sigmoidal transformations}

We now consider the effect of applying a sigmoidal transformation of order $r$ (see Section 3) to the integral I $f(\cdot ; \lambda)$, see (143). On writing

$$
x=\gamma_{r}(t), \quad \lambda=\gamma_{r}(s),
$$

where $0<s<1$, we may, on using the usual rules for the change of variable of integration, rewrite $\operatorname{If}(\cdot ; \lambda)$ as

$$
I f(\cdot ; \lambda)=I F_{r}(\cdot ; s)=f_{0}^{1} \frac{G_{r}(t, s) d t}{t-s} .
$$

The function $F_{r}$ is defined on $\bar{\Omega} \times \Omega$ by

$$
F_{r}(t ; s):= \begin{cases}\frac{G_{r}(t, s)}{t-s}, & t \neq s, \\ 0, & t=s,\end{cases}
$$


say, compare with (142), where the function $G_{r}$ is defined on $\bar{\Omega} \times \Omega$ by

$$
G_{r}(t ; s):= \begin{cases}\frac{t-s}{\gamma_{r}(t)-\gamma_{r}(s)} \phi\left(\gamma_{r}(t)\right) \gamma_{r}^{\prime}(t), & t \neq s \\ \phi(\lambda), & t=s .\end{cases}
$$

We must first determine a weighted Sobolev space for the function $G_{r}(\cdot ; s)$.

Theorem 24 Consider the case $\phi \in W_{p, \alpha}^{(N)}(\Omega)$ for $1 \leq p \leq \infty$, a real and $N \in \mathbb{N}_{0}$. If $\gamma_{r}$ is a sigmoidal transformation of order $r \geq 1$ then $G_{r}(\cdot ; s) \in W_{p, \alpha r+(r-1) / q-\epsilon}^{(N)}(\Omega)$ for any $\epsilon>0$ with

$$
\left\|G_{r}(\cdot ; s)\right\|_{p, \alpha r+(r-1) / q-\epsilon, N} \leq \frac{c\|\phi\|_{p, \alpha, N}}{\lambda(1-\lambda)},
$$

where $c$ is independent of $\lambda$ and $\phi$.

Proof: On $\bar{\Omega} \times \Omega$ let us define a function

$$
\Phi_{r}(x ; \lambda):= \begin{cases}\frac{\gamma_{r}^{-1}(x)-\gamma_{r}^{-1}(\lambda)}{x-\lambda} \phi(x), & x \neq \lambda, \\ \left(\gamma_{r}^{-1}\right)^{\prime}(\lambda) \phi(\lambda), & x=\lambda .\end{cases}
$$

Since $\left(\gamma_{r}^{-1}\right)^{\prime}(\lambda)=1 / \gamma_{r}^{\prime}\left(\gamma_{r}^{-1}(\lambda)\right)$ it is readily shown that

$$
G_{r}(t ; s)=\Phi_{r}\left(\gamma_{r}(t) ; \lambda\right) \gamma_{r}^{\prime}(t) .
$$

In order to use Theorem 10 to determine an appropriate space for $G_{r}$, we must first determine a space for $\Phi_{r}$. First, let us define

$$
\Gamma_{r}(x ; \lambda):= \begin{cases}\frac{\gamma_{r}^{-1}(x)-\gamma_{r}^{-1}(\lambda)}{x-\lambda}, & x \neq \lambda, \\ \left(\gamma_{r}^{-1}\right)^{\prime}(\lambda), & x=\lambda .\end{cases}
$$

The function $\gamma_{r}^{-1}$ is infinitely differentiable on $\Omega$ and $\gamma_{r}^{-1}(x)=\mathcal{O}\left(x^{1 / r}\right)$ near $x=0$ and $\gamma_{r}^{-1}(x)=1+\mathcal{O}\left((1-x)^{1 / r}\right)$ near $x=1$. It follows that, for any 
$\epsilon>0$ and $N_{1} \in \mathbb{N}_{0}$, we will have $\gamma_{r}^{-1} \in W_{\infty,-\epsilon}^{\left(N_{1}\right)}(\Omega)$. By Theorem 26, for $0<\epsilon<1$ we have that $\Gamma_{r}(\cdot ; \lambda) \in W_{\infty,-\epsilon}^{\left(N_{1}-1\right)}(\Omega)$ with

$$
\left\|\Gamma_{r}(\cdot ; \lambda)\right\|_{\infty,-\epsilon, N_{1}-1} \leq \frac{c\left\|\gamma_{r}^{-1}\right\|_{\infty,-\epsilon, N_{1}}}{\lambda(1-\lambda)}
$$

where $c$ is independent of $\lambda$. Since $\Phi_{r}(\cdot ; \lambda)=\phi \Gamma_{r}(\cdot ; \lambda)$ it follows from Theorem 8 that if we choose $N_{1}=N+1$ then, since $\phi \in W_{p, \alpha}^{(N)}(\Omega)$, we shall have $\Phi_{r}(\cdot ; \lambda) \in W_{p, \alpha-\epsilon}^{(N)}(\Omega)$ with

$$
\left\|\Phi_{r}(\cdot ; \lambda)\right\|_{p, \alpha-\epsilon, N} \leq \frac{c\|\phi\|_{p, \alpha, N}}{\lambda(1-\lambda)}
$$

where $c$ is now independent of $\lambda$ and $\phi$. From (238) and Theorem 10 it follows that $G_{r}(\cdot ; s) \in W_{p, \alpha r+(r-1) / q-\epsilon}^{(N)}(\Omega)$, for any $\epsilon>0$, and (236) follows at once.

We are now in a position to consider the quadrature rule and its truncation error after a sigmoidal transformation has been applied. Let us recapitulate on the quadrature rule we have obtained so far. Applying (223) to the function $F_{r}$, see (234), we have

$$
\begin{aligned}
I f(\cdot ; \lambda)=I F_{r}(\cdot ; s) & =Q_{m}^{[\nu]} F_{r}(\cdot ; s)-S_{m}^{[\nu]} F_{r}(\cdot ; s)+E_{m}^{[\nu]} F_{r}(\cdot ; s) \\
& =: Q_{m}^{[\nu, r]} f(\cdot ; \lambda)-S_{m}^{[\nu, r]} f(\cdot ; \lambda)+E_{m}^{[\nu, r]} f(\cdot ; \lambda),
\end{aligned}
$$

say. Thus we have

$$
Q_{m}^{[\nu, r]} f(\cdot ; \lambda)=Q_{m}^{[\nu]} F_{r}(\cdot ; s)= \begin{cases}\frac{1}{m} \sum_{j=0}^{m-1} F_{r}\left(\left(j+t_{\nu}\right) / m ; s\right), & -1<\nu<1, \\ \frac{1}{m} \sum_{j=0}^{m} F_{r}(j / m ; s), & \nu=1 .\end{cases}
$$

Again, we note that these sums are always defined since, from (234), we have defined $F_{r}$ to be zero when $t=s$. 
From (235) and the use of l'Hôpital's rule it is straightforward to show that

$$
G_{r}^{\prime}(s ; s)=\frac{2\left(\gamma_{r}^{\prime}(s)\right)^{2} \phi^{\prime}(\lambda)+\gamma_{r}^{\prime \prime}(s) \phi(\lambda)}{2 \gamma_{r}^{\prime}(s)} .
$$

From (221), (235) and (242) we have

$$
S_{m}^{[\nu, r]} f(\cdot ; \lambda)=S_{m}^{[\nu]} F_{r}(\cdot ; s)= \begin{cases}\pi \phi(\lambda) \cot \left(\pi\left(t_{\nu}-m s\right)\right), & m s-t_{\nu} \notin \mathbb{Z}, \\ -G_{r}^{\prime}(s ; s) / m, & m s-t_{\nu} \in \mathbb{Z},\end{cases}
$$

where $G_{r}^{\prime}(s ; s)$ is defined in (244).

We are now in a position to state the principal result of this section.

Theorem 25 Let $\gamma_{r}$ be a sigmoidal transformation of order $r>1$. Suppose $\phi \in W_{p, \alpha}^{(N)}(\Omega)$ where $1 \leq p \leq \infty$ and $n_{r}, N \in \mathbb{N}$ are such that

$$
n_{r}<r(\alpha+1 / q)-\epsilon<n_{r}+1 \leq N .
$$

For $m \in \mathbb{N}$ such that $m \geq\left[n_{r} / 2\right]+1$ and $\lambda \in \Omega$ we have

$$
I f(\cdot ; \lambda)=f_{0}^{1} \frac{\phi(x) d x}{x-\lambda}=Q_{m}^{[\nu, r]} f(\cdot ; \lambda)-S_{m}^{[\nu, r]} f(\cdot ; \lambda)+E_{m}^{[\nu, r]} f(\cdot ; \lambda)
$$

where $Q_{m}^{[\nu, r]}$ and $S_{m}^{[\nu, r]}$ are defined in (243)-(245). The truncation error $E_{m}^{[\nu, r]}$ is such that

$$
\left|E_{m}^{[\nu, r]} f(\cdot ; \lambda)\right| \leq \frac{c\|\phi\|_{p, \alpha, N}}{(\lambda(1-\lambda))^{1+2 / r} m^{r(\alpha+1 / q)-\epsilon}}
$$

where $\epsilon$ is an arbitrarily small positive number and $c$ is independent of $\lambda$, $m$ and $\phi$. 
Proof: This follows by Theorem 23 where we replace $f(\cdot ; \lambda)$ by $F_{r}(\cdot ; s)$ and $\phi$ by $G_{r}(\cdot ; s)$. If, in (224) applied to $G_{r}(\cdot ; s)$, we replace the exponent " $n+2-(\alpha+1 / q)$ " by 2 we have

$$
\left|E_{m}^{[\nu, r]} f(\cdot ; \lambda)\right| \leq \frac{c\left\|G_{r}(\cdot ; s)\right\|_{p, \alpha r+(r-1) / q-\epsilon, N}}{(s(1-s))^{2} m^{r(\alpha+1 / q)-\epsilon}},
$$

where $\epsilon$ is arbitrarily small and positive. On recalling (92) and (232) we have, since $\gamma_{r}^{-1}(t)=\mathcal{O}\left(t^{1 / r}\right)$ near $t=0$ and $1-\gamma_{r}^{-1}(t)=\mathcal{O}\left((1-t)^{1 / r}\right)$ near $t=1$, that there exist constants $c_{1}$ and $c_{2}$ say, such that

$$
c_{1}(\lambda(1-\lambda))^{1 / r} \leq s(1-s)=\gamma_{r}^{-1}(\lambda)\left(1-\gamma_{r}^{-1}(\lambda)\right) \leq c_{2}(\lambda(1-\lambda))^{1 / r} .
$$

From (236), (249) and (250) we recover (248) and the theorem is proved.

Interestingly, in (248), note the dependence of the truncation error on $\lambda$ and we see that for all $r>1$, we have $1+2 / r<3$. The rate of convergence of the truncation error is $\mathcal{O}\left(1 / m^{r(\alpha+1 / q)-\epsilon}\right)$ which is almost that for the ordinary integral as given in (140). The author has been unable to put $\epsilon$ equal to zero, although it can be assumed to be arbitrarily small and positive.

\section{Conclusion}

In this paper we have introduced the sigmoidal-trapezoidal quadrature rule for the approximate evaluation of both ordinary and Cauchy principal value integrals. The paper is then concerned with establishing the rates of convergence of these quadrature rules given that the integrand is in a particular weighted Sobolev space. We have found comparable rates of convergence for both ordinary (see Theorem 15) and Cauchy principal value (see Theorem 25) integrals. The ultimate aim of this analysis is its application to the approximate solution of various integral equations. Some results have already been made in this direction; see Kress [7], Elliott [3] and Elliott and Prössdorf [6]. 
An unpublished report by Duduchava, Elliott and Wendland [2] also makes considerable progress in this direction.

What we have not done in this paper is to give any numerical results for the quadrature of explicit functions. This will have to await another occasion; this paper is long enough as it is. In this regard the paper by Weideman [9] should be of considerable relevance.

Acknowledgment: The author is extremely grateful to an unknown referee who made some excellent constructive comments on the first draft of this paper. In particular, the referee suggested Theorem 16 which simplified much of the earlier analysis. This, in turn, inspired the author to review Section 5 thoroughly. Theorems 23 and 25 are now much improved versions of the corresponding theorems which appeared in the first draft.

\section{A Appendix}

In this appendix we state and prove two theorems which are useful in Section 5 where we consider Cauchy principal value integrals. It is convenient to put these into an appendix and quote them as required rather than interrupt the arguments in Section 5, which is long enough anyway. In these theorems the function $\Psi$ is defined on $\bar{\Omega} \times \Omega$ by

$$
\Psi(x ; \lambda):= \begin{cases}\frac{\psi(x)-\psi(\lambda)}{x-\lambda}, & x \neq \lambda, \\ \psi^{\prime}(\lambda), & x=\lambda .\end{cases}
$$

Theorem 26 Suppose $\psi \in W_{\infty, \beta}^{(N)}(\Omega)$ where $N \in \mathbb{N}$ and $0<\beta+1<1$. Then 
$\Psi(\cdot ; \lambda) \in W_{\infty, \beta}^{(N-1)}(\Omega)$ with

$$
\|\Psi(\cdot ; \lambda)\|_{\infty, \beta, N-1} \leq \frac{c\|\psi\|_{\infty, \beta, N}}{\lambda(1-\lambda)},
$$

where $c$ is a positive constant independent of $\lambda$ and $\psi$.

Proof: For $j=0(1)(N-1)$ and given $\lambda \in \Omega$, define $I_{j}(\lambda)$ by

$$
I_{j}(\lambda):=\max _{0 \leq x \leq 1}(x(1-x))^{j-\beta}\left|\Psi^{(j)}(x ; \lambda)\right| .
$$

We need to show that $I_{j}(\lambda)$ is finite for $j=0(1)(N-1)$.

Suppose first that $0 \leq x \leq \lambda$. From (251) we have

$$
\Psi(x ; \lambda)=\int_{0}^{1} \psi^{\prime}(x+(\lambda-x) u) d u,
$$

so that for $j=0(1)(N-1)$ we have

$$
\Psi^{(j)}(x ; \lambda)=\int_{0}^{1}(1-u)^{j} \psi^{(j+1)}(x+(\lambda-x) u) d u .
$$

From (15),

$$
\left|\psi^{(j)}(x+(\lambda-x) u)\right| \leq\|\psi\|_{\infty, \beta, N}\left(\frac{1}{x+(\lambda-x) u}+\frac{1}{1-x-(\lambda-x) u}\right)^{j+1-\beta}
$$

so that from (255)

$\left|\Psi^{(j)}(x ; \lambda)\right| \leq\|\psi\|_{\infty, \beta, N} \int_{0}^{1}(1-u)^{j}\left(\frac{1}{x+(\lambda-x) u}+\frac{1}{1-x-(\lambda-x) u}\right)^{j+1-\beta} d u$ 
From Hölder's inequality for sums we obtain

$$
\left|\Psi^{(j)}(x ; \lambda)\right| \leq c\|\psi\|_{\infty, \beta, N}\left(\Phi_{j}(x ; \lambda)+\Phi_{j}(1-x ; 1-\lambda)\right),
$$

say, $c$ being independent of $x, \lambda$ and $\psi$, and where we define

$$
\Phi_{j}(x ; \lambda):=\int_{0}^{1} \frac{(1-u)^{j} d u}{(x+(\lambda-x) u)^{j+1-\beta}} .
$$

In order to obtain an upper bound for $\Phi_{j}(\cdot ; \lambda)$ on $[0, \lambda]$ we first identify it as a hypergeometric function. From Abramowitz and Stegun [1, §15.3.1] we find, on replacing $u$ by $1-u$ in the integrand of (259), that

$$
\Phi_{j}(x ; \lambda)=\frac{1}{\lambda^{j+1-\beta}(j+1)} \times F(j+1-\beta, j+1 ; j+2 ;(\lambda-x) / \lambda) .
$$

On applying the linear transformation $[1, \S 15.3 .3]$, we obtain

$$
\Phi_{j}(x ; \lambda)=\frac{x^{\beta-j}}{\lambda(j+1)} \times F(1+\beta, 1 ; j+2 ;(\lambda-x) / \lambda) .
$$

For $0 \leq x \leq \lambda$, the argument of this hypergeometric function lies in the interval $[0,1]$. Again, from $[1, \S 15.1 .1]$, since $j-\beta>0$ for $j=0(1)(N-1)$, it follows that the series expansion for this hypergeometric function is absolutely convergent on the unit circle. Hence, for $0<x \leq \lambda$, we have that

$$
\Phi_{j}(x ; \lambda) \leq \frac{c x^{\beta-j}}{\lambda},
$$

for some $c$ independent of $x$ and $\lambda$.

Consider now $\Phi_{j}(1-x ; 1-\lambda)$ which, from (260), may be written as $\Phi_{j}(1-x ; 1-\lambda)=\frac{1}{(1-\lambda)^{j+1-\beta}(j+1)} F(j+1-\beta, j+1 ; j+2 ;-(\lambda-x) /(1-\lambda))$. 
On applying the linear transformation $[1, \S 15.3 .5]$ we have

$$
\Phi_{j}(1-x ; 1-\lambda)=\frac{(1-x)^{-j-1}}{(1-\lambda)^{-\beta}(j+1)} F(j+1,1+\beta ; j+2 ;(\lambda-x) /(1-x)) .
$$

For $0 \leq x \leq \lambda$ we see that $0 \leq(\lambda-x) /(1-x) \leq \lambda<1$ and, since $-\beta>0$, it follows from $[1, \S 15.1 .1]$ that the series expansion for this hypergeometric function converges absolutely on the unit circle. Hence there exists a constant $c$, independent of $\lambda$ and $x$, such that

$$
\Phi_{j}(1-x ; 1-\lambda) \leq \frac{c(1-x)^{-j-1}}{(1-\lambda)^{-\beta}} .
$$

From (258), (262) and (265) it follows that for $0<x \leq \lambda$

$$
\left|\Psi^{(j)}(x ; \lambda)\right| \leq c\|\psi\|_{\infty, \beta, N}\left(\frac{x^{\beta-j}}{\lambda}+\frac{(1-x)^{-1-j}}{(1-\lambda)^{-\beta}}\right) .
$$

We may now proceed similarly for the interval $\lambda \leq x \leq 1$. Again we have an upper bound on $\left|\Psi^{(j)}(x ; \lambda)\right|$ given by $(258)$ and $(260)$. If, to the hypergeometric function in (260), we apply the linear transformation [1, 115.3 .5$]$ we find

$$
\Phi_{j}(x ; \lambda)=\frac{x^{-j-1}}{\lambda^{-\beta}(j+1)} F(j+1,1+\beta ; j+2 ; 1-\lambda / x) .
$$

For $\lambda \leq x \leq 1$ we see that the argument of this hypergeometric function lies in the interval $[0,1-\lambda]$ and, since $-\beta>0$, we have that its series expansion converges absolutely on the unit circle. Consequently, for $\lambda \leq x \leq 1$ we have

$$
\Phi_{j}(x ; \lambda) \leq \frac{c x^{-j-1}}{\lambda^{-\beta}},
$$

where $c$ is independent of $\lambda$ and $x$. Finally we consider $\Phi_{j}(1-x ; 1-\lambda)$ for $\lambda \leq x \leq 1$. If to the hypergeometric function in (263) we apply the linear transformation $[1, \S 15.3 .3]$ we find

$$
\Phi_{j}(1-x ; 1-\lambda)=\frac{(1-x)^{\beta-j}}{(1-\lambda)(j+1)} F(1+\beta, 1 ; j+2 ;-(\lambda-x) /(1-x)) .
$$


Again, for $\lambda \leq x \leq 1$, the argument of this hypergeometric function lies in $[0,1]$ and, since $-\beta<0$, its series expansion converges absolutely on the unit circle. Hence there exists a constant $c$, independent of $\lambda$ and $x$, such that

$$
\Phi_{j}(1-x ; 1-\lambda) \leq \frac{c(1-x)^{\beta-j}}{(1-\lambda)},
$$

for $\lambda \leq x \leq 1$. From (258), (265) and (270) we have, for $\lambda \leq x \leq 1$, that

$$
\left|\Psi^{(j)}(x ; \lambda)\right| \leq c\|\psi\|_{\infty, \beta, N}\left(\frac{x^{-j-1}}{\lambda^{-\beta}}+\frac{(1-x)^{\beta-j}}{(1-\lambda)}\right) .
$$

Returning to (253), we put an upper bound on $I_{j}(\lambda)$ for $j=0(1)(N-1)$. We have

$$
\begin{aligned}
& I_{j}(\lambda)=\max \left\{\max _{0 \leq x \leq \lambda}\left((x(1-x))^{j-\beta}\left|\Psi^{(j)}(x ; \lambda)\right|\right),\right. \\
& \left.\max _{\lambda \leq x \leq 1}\left((x(1-x))^{j-\beta}\left|\Psi^{(j)}(x ; \lambda)\right|\right)\right\} .
\end{aligned}
$$

From (266) and (271) we find

$$
\begin{aligned}
I_{j}(\lambda) \leq c\|\psi\|_{\infty, \beta, N} \max \{ & \max _{0 \leq x \leq \lambda}\left(\frac{(1-x)^{j-\beta}}{\lambda}+\frac{x^{j-\beta}(1-x)^{-1-\beta}}{(1-\lambda)^{-\beta}}\right), \\
& \left.\max _{\lambda \leq x \leq 1}\left(\frac{x^{-1-\beta}(1-x)^{j-\beta}}{\lambda^{-\beta}}+\frac{x^{j-\beta}}{(1-\lambda)}\right)\right\} \cdot(273)
\end{aligned}
$$

Since $j-\beta>0$ for $j=0(1)(N-1)$ and since $1+\beta>0$ we see from (273) that

$$
I_{j}(\lambda) \leq \frac{c\|\psi\|_{\infty, \beta, N}}{\lambda(1-\lambda)}<\infty .
$$

Consequently $\Psi(\cdot ; \lambda) \in W_{\infty, \beta}^{(N-1)}(\Omega)$ with the norm of $\Psi$ satisfying (252). 
Comment: We could have allowed $\beta=0$ in Theorem 26 but some modification would have been needed to the statement of the theorem. We have decided not to pursue this case here.

We now come to the second of our theorems.

Theorem 27 Suppose $\psi \in W_{p, \beta}^{(N)}(\Omega)$ where $1 \leq p<\infty, 0<\beta+1 / q<1$ and $\mathbb{N} \ni N \geq 2$. Then, for any $\epsilon>0, \Psi(\cdot ; \lambda) \in W_{p, \beta-\epsilon}^{(N-2)}(\Omega)$ with

$$
\|\Psi(\cdot ; \lambda)\|_{p, \beta-\epsilon, N-2} \leq \frac{c\|\psi\|_{p, \beta, N}}{\lambda(1-\lambda)}
$$

$c$ being independent of $\psi$ and $\lambda$.

Proof: By Theorem $6(\mathrm{a})$, since $\psi \in W_{p, \beta}^{(N-1)}(\Omega)$ then we shall also have $\psi \in W_{\infty, \beta-1 / p}^{(N-1)}(\Omega)$ with

$$
\|\psi\|_{\infty, \beta-1 / p, N-1} \leq c\|\psi\|_{p, \beta, N},
$$

$c$ being independent of $\psi$. By Theorem 26, since $\beta-1 / p+1<1$, it follows that $\Psi(\cdot ; \lambda) \in W_{p, \beta-1 / p}^{(N-2)}(\Omega)$ with

$$
\|\Psi(\cdot ; \lambda)\|_{\infty, \beta-1 / p, N-2} \leq \frac{c\|\psi\|_{\infty, \beta-1 / p, N-1}}{\lambda(1-\lambda)} \leq \frac{c\|\psi\|_{p, \beta, N}}{\lambda(1-\lambda)}
$$

by (276), where $c$ is independent of both $\psi$ and $\lambda$. By Theorem $6(\mathrm{~b})$, since $\Psi(\cdot ; \lambda) \in W_{\infty, \beta-1 / p}^{(N-2)}(\Omega)$ it follows that $\Psi(\cdot ; \lambda) \in W_{p, \beta-\epsilon}^{(N-2)}(\Omega)$ for any $\epsilon>0$ and furthermore from (277) and (58) it follows that (275) is satisfied and the theorem is proved. 


\section{References}

[1] M. Abramowitz and I. A. Stegun, Handbook of Mathematical Functions, Dover Publications Inc., New York, 1972. E30, E31, E44, $\mathrm{E} 45, \mathrm{E} 47, \mathrm{E} 50, \mathrm{E} 64, \mathrm{E} 65$

[2] R. Duduchava, D. Elliott and W. L. Wendland The spline collocation method for Mellin convolution equations, Technical Report, University of Stuttgart, April,1996. E62

[3] D. Elliott, The cruciform crack problem and sigmoidal transformations, Mathematical Methods in the Applied Sciences, 20, pp.121-132, 1997. E61

[4] D. Elliott, The Euler-Maclaurin formula revisited, J. Aust. Maths. Soc. B(E), 40, pp.E27-E76, 1998.

http://anziamj.austms.org.au/V40/E005 E3, E7, E29, E30

[5] D. Elliott, Sigmoidal transformations and the trapezoidal rule, J. Aust. Maths. Soc. B(E), 40, pp.E77-E137, 1998.

http://anziamj.austms.org.au/V40/E006 E3, E24, E36

[6] D. Elliott and S. Prössdorf, An algorithm for the approximate solution of integral equations of Mellin type, Numer. Math., 70, pp.427-452, 1995. E61

[7] R. Kress, A Nyström method for boundary integral equations in domains with corners, Numer. Math., 58, pp.145-161, 1990. E3, E7, E61

[8] J. N. Lyness, The Euler-Maclaurin expansion for the Cauchy principal value integral, Numer. Math., 46, pp. 611-622, 1985. E44, E46, E55, E57 
[9] J. A. C. Weideman, Numerical Integration of Periodic Functions: A Few Examples, The American Mathematical Monthly, 109, pp.21-36, 2002. E62 